\title{
Towards Feynman rules for Mellin amplitudes in AdS/CFT
}

\author{
Miguel F. Paulos \\ Laboratoire de Physique Théorique et Hautes Energies, CNRS UMR 7589, \\ Université Pierre et Marie Curie, \\ 4 place Jussieu, 75252 Paris Cedex 05, France \\ E-mail: mpaulos@lpthe.jussieu.fr
}

ABSTRACT: We investigate the use of the embedding formalism and the Mellin transform in the calculation of tree-level conformal correlation functions in AdS/CFT. We evaluate 5- and 6-point Mellin amplitudes in $\phi^{3}$ theory and even a 12-pt diagram in $\phi^{4}$ theory, enabling us to conjecture a set of Feynman rules for scalar Mellin amplitudes. The general vertices are given in terms of Lauricella generalized hypergeometric functions. We also show how to use the same combination of Mellin transform and embedding formalism for amplitudes involving fields with spin. The complicated tensor structures which usually arise can be written as certain operators acting as projectors on much simpler index structures essentially the same ones appearing in a flat space amplitude. Using these methods we are able to evaluate a four-point current diagram with current exchange in Yang-Mills theory.

KeYwords: AdS-CFT Correspondence, Conformal and W Symmetry, Gauge-gravity correspondence

ARXIV EPRINT: 1108.3077 


\section{Contents}

1 Introduction $\quad 2$

1.1 Summary of results 4

2 Preliminaries $\quad 7$

2.1 Embedding formalism $\quad 7$

2.2 Boundary-bulk propagators 8

$\begin{array}{lll}2.3 & \text { Bulk-to-bulk propagators } & 10\end{array}$

3 Warm-up: 3 and 4-point scalar correlation functions 11

3.13 -point vertex 11

3.2 4-point exchange diagram 12

4 Scalar higher-point amplitudes $\quad 16$

4.1 5-point amplitude 16

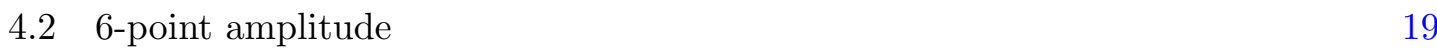

4.3 Outline of the 12-point amplitude calculation 22

5 Conformal invariance of index structure 23

6 Current amplitudes $\quad 25$

$\begin{array}{lll}6.1 & \langle\boldsymbol{J O O}\rangle & \text { correlator }\end{array}$

6.2 Current three-point amplitude 26

$\begin{array}{lll}6.3 & \text { Scalar 4-point with current exchange } & 27\end{array}$

6.4 Current 4-point amplitude 30

7 Discussion and outlook $\quad 32$

$\begin{array}{ll}\text { A Some integrals } & 34\end{array}$

B The Symanzik star formula $\quad 35$

C Details on the calculation of the six-point amplitude 36

D Index structure of current four-point function contact diagram $\quad 37$ 


\section{Introduction}

Witten diagrams [1] provide us with the means for calculating correlation functions [2] of strongly coupled conformal field theories with a gravity dual [3, 4]. However, in spite of significant progress [5-8], such calculations are in general quite cumbersome to perform. As it stands, the state of the art is the computation of four point functions involving different kinds of exchanged fields in type IIB supergravity ${ }^{1}$ [12-15], and a stress-tensor three point function [16]. The latter constitutes an especially heroic effort, due to the complicated tensor structures required for conformal invariance of the three-point function $[17,18]$.

Such calculations are usually performed in coordinate space. An obvious question is whether changing basis could lead to simplifications. The first guess is momentum space, but this doesn't lead to any major simplifications - perhaps the reason is simply that such a transformation does not take into account the symmetries of AdS space, but only of its boundary. As it turns out that a more appropriate basis does exist: instead of the Fourier transform one should really be working with the Mellin transform [19-21]

The Mellin transform is very natural from a conformal field theory perspective. To see this consider the four-point function of a scalar fields $\mathcal{O}_{i}$ of conformal dimension $\Delta_{i}$. By using the OPE in the 12 channel say, we can write

$$
\begin{aligned}
& \left\langle\mathcal{O}_{\Delta_{1}}\left(x_{1}\right) \mathcal{O}_{\Delta_{1}}\left(x_{2}\right) \mathcal{O}_{\Delta_{1}}\left(x_{3}\right) \mathcal{O}_{\Delta_{1}}\left(x_{4}\right)\right\rangle \\
& \quad=\int \frac{\mathrm{d} c}{2 \pi i} g^{(12)(34)}(c) \int \mathrm{d}^{d} x\left\langle\mathcal{O}\left(x_{1}\right) \mathcal{O}\left(x_{2}\right) \phi_{h+c}(x)\right\rangle\left\langle\phi_{h-c}(x) \mathcal{O}\left(x_{3}\right) \mathcal{O}\left(x_{4}\right)\right\rangle+\ldots
\end{aligned}
$$

where the ... represent contributions of fields with spin appearing in the OPE, $\phi_{h \pm c}$ is a scalar field of unphysical dimension $h \pm c$, and $g^{(12)(34)}(c)$ contains the information about which scalar fields appear in the OPE, through its pole structure. The three point functions appearing above are uniquely fixed by conformal symmetry, say

$$
\left\langle\mathcal{O}_{\Delta_{1}}\left(x_{1}\right) \mathcal{O}_{\Delta_{2}}\left(x_{2}\right) \mathcal{O}_{\Delta_{3}}(x)\right\rangle=C_{\Delta_{1}, \Delta_{2}, \Delta_{3}} \prod_{i<j}^{3}\left(x_{i}-x_{j}\right)^{-2 \tilde{\Delta}_{i j}}
$$

with e.g. $\tilde{\Delta}_{12}=\frac{1}{2}\left(\Delta_{1}+\Delta_{2}-\Delta_{3}\right)$ and $C_{\Delta_{1}, \Delta_{2}, \Delta_{3}}$ is a constant which contains information about the dynamics. Therefore the integral becomes

$$
\begin{aligned}
& \left\langle\mathcal{O}\left(x_{1}\right) \mathcal{O}\left(x_{2}\right) \mathcal{O}\left(x_{3}\right) \mathcal{O}\left(x_{4}\right)\right\rangle=\int \frac{\mathrm{d} c}{2 \pi i} g^{(12)(34)}(c) C_{\Delta_{1}, \Delta_{2}, h+c} C_{\Delta_{3}, \Delta_{4}, h-c} \\
& \quad\left(x_{1}-x_{2}\right)^{-\left(\Delta_{1}+\Delta_{2}-(h+c)\right)}\left(x_{3}-x_{4}\right)^{-\left(\Delta_{3}+\Delta_{4}-(h+c)\right)} \int \mathrm{d}^{d} x \prod_{i=1}^{4}\left(x-x_{i}\right)^{-\delta_{i}}+\ldots
\end{aligned}
$$

with

$$
\begin{array}{ll}
\delta_{1}=\frac{1}{2}\left(\Delta_{1}+h+c-\Delta_{2}\right), & \delta_{2}=\frac{1}{2}\left(\Delta_{2}+h+c-\Delta_{1}\right), \\
\delta_{3}=\frac{1}{2}\left(\Delta_{3}+h-c-\Delta_{4}\right), & \delta_{2}=\frac{1}{2}\left(\Delta_{4}+h-c-\Delta_{3}\right),
\end{array}
$$

\footnotetext{
${ }^{1}$ See also the works [9-11] where correlators of currents are calculated in certain limits.
} 
To perform the $x$ integral in (1.3) the standard procedure is to introduce Schwinger parameters to exponentiate the denominators. The $x$ integration becomes trivial, and the Schwinger integrations can be performed via Symanzik's star formula [22], as we discuss in appendix B. The net result is that

$$
\pi^{-d / 2} \int \mathrm{d}^{d} x \prod_{i=1}^{4}\left(x-x_{i}\right)^{-\delta_{i}} \Gamma\left(\delta_{i}\right)=\int \mathrm{d} \delta_{i j} \prod_{i<j}^{4} \Gamma\left(\tilde{\delta}_{i j}\right)\left(x_{i}-x_{j}\right)^{-2 \tilde{\delta}_{i j}}
$$

where the $n(n-3) / 2$ independent parameters $\tilde{\delta}_{i j}$ satisfy the constraints $\sum_{i \neq j} \tilde{\delta}_{i j}=\delta_{j}$. In this way, we have passed from integrations in coordinate space to integrations in the Mellin space.

Generically, any conformal field theory correlation function of scalars with dimensions $\Delta_{i}$ can be written in the Mellin representation as [19]:

$$
A\left(x_{1}, x_{2}, \ldots, x_{n}\right)=\frac{\mathcal{N}}{(2 \pi i)^{\frac{1}{2} n(n-3)}} \int \mathrm{d} \delta_{i j} M\left(\delta_{i j}\right) \prod_{i<j}^{n} \Gamma\left(\delta_{i j}\right)\left(x_{i}-x_{j}\right)^{-2 \delta_{i j}} .
$$

The normalization constant $\mathcal{N}$ will be fixed later. The object $M\left(\delta_{i j}\right)$ is the Mellin amplitude, which depends on a set of $n(n-3) / 2$ parameters $\delta_{i j}$ equal in number to the number of independent cross-ratios. ${ }^{2}$ These parameters satisfy the constraints

$$
\sum_{j} \delta_{i j}=\Delta_{i}, \quad \delta_{i i}=0
$$

which may be solved by introducing a set of $d$-dimensional vectors $k_{i}$ satisfying

$$
-k_{i}^{2}=\Delta_{i}, \quad \sum_{i} k_{i}=0
$$

in terms of which $\delta_{i j}=k_{i} \cdot k_{j}$. It is also useful to introduce the "Mandelstam invariants"

$$
s_{i_{1} i_{2} \ldots i_{p}}=-\left(\sum_{m=1}^{p} k_{i_{m}}\right)^{2}=\sum_{m=1}^{p} \Delta_{i_{m}}-2 \sum_{i_{k}<i_{l}} \delta_{i_{k} j_{l}},
$$

which imply for instance $s_{i j}=-\left(k_{i}+k_{j}\right)^{2}=\Delta_{i}+\Delta_{j}-2 \delta_{i j}$.

Mellin amplitudes have very simple analytic properties. The scalar four-point function for instance, has an infinite set of simple poles in the $s$-channel at $s_{12}=\Delta_{k}-s_{k}+2 n$, where $\Delta_{k}, s_{k}$ are the conformal dimension and spin respectively of a field appearing in the OPE, and $n$ is a positive integer. The residues of the satellite poles, that is those with $n \neq 0$, are completely fixed by conformal symmetry in terms of the leading $n=0$ pole. Further, validity of the OPE requires factorisation: the residue of the leading pole splits into the product of two factors, one pertaining only to fields 12 and the other to fields 34 .

In the paper [21], the Mellin formalism was used to study CFT correlation functions computed in the AdS/CFT context, with promising results. For instance, contact interactions have simply polynomials as their Mellin amplitudes, in contrast to the complicated

\footnotetext{
${ }^{2}$ As long as this number is smaller than $n \times d$, the number of maximally independent components of $n$-dimensional vectors.
} 
$D$-functions which appear in coordinate space. Even the dreaded stress-tensor exchange diagram reduces to a simple rational function for the case of minimally coupled massless scalars. The simple analytic properties of Mellin amplitudes also make clear which operators are propagating throughout a given Witten diagram: double-trace operators corresponding to the fusion of external legs are captured by the explicit gamma functions in the Mellin representation, whereas single-trace operators and their descendants corresponding to internal lines or bulk-to-bulk propagators, appear as simple poles of the Mellin amplitude,.

In this paper we continue to investigate the properties of AdS/CFT correlation functions in the Mellin representation. We shall do this on two fronts. Firstly by evaluating higher point amplitudes in purely scalar theory, that is, where no other fields other than scalars propagate in a Witten diagram. Secondly by computing correlation functions of operators with spin such as currents and stress-tensors. In both cases it will be invaluable to use the embedding formalism [23-25]. The main idea is to think of $A d S_{d+1}$ space as embedded in flat Minkowski space $M_{d+2}$, with metric $\eta^{M N}$. AdS coordinate vectors $X^{M}$ satisfy $X \cdot X=-R^{2}$ whereas AdS boundary coordinates $P^{M}$ are defined by $P^{2}=0$, $P \simeq \alpha P, \alpha>0$. With the two-pronged approach of using embedding formalism and Mellin transforms, the computation of correlation functions simplifies dramatically.

\subsection{Summary of results}

An intriguing possibility raised by the work of [21] is the existence of Feynman rules for Mellin amplitudes. Indeed, the Mellin amplitude for a scalar four point function in $\phi^{3}$ theory takes the simple form

$$
M_{4} \simeq \sum_{n=0}^{+\infty}\left(\frac{V_{\Delta, n}^{2}}{s_{12}-\Delta-2 n}+\frac{V_{\Delta, n}^{2}}{s_{13}-\Delta-2 n}+\frac{V_{\Delta, n}^{2}}{s_{14}-\Delta-2 n}\right)
$$

The vertex $V_{\Delta, n}$ essentially describes the three point function of two scalars and a descendant field at level $n$. The above is remarkably similar to a flat space scattering amplitude, and indeed it becomes one for high enough energies as compared to the dimensions $\Delta$. In this work we shall present strong evidence that at least for scalar theory, it is possible to write down a set of Feynman rules for Mellin amplitudes. More precisely, we compute 5-pt, 6-pt and even a 12-pt diagram in scalar theory and check that the rules hold. These calculations also allow us to read off the vertices $V$ when more than one descendant fields are involved. In $\phi^{3}$ theory we need at least three internal lines (bulk-to-bulk propagators) to see three descendant fields interacting, and in $\phi^{4}$ theory we need four such lines. Our computations are consistent with the existence of a set of Feynman rules for Mellin diagrams, which are given in the following.

Conjecture (Feynman rules for Mellin amplitudes). Consider a tree-level Witten diagram involving only scalar fields, consisting of a set of external (bulk to boundary) and internal (bulk to bulk) lines, and vertices connecting them. The corresponding Mellin amplitude is constructed as follows: 
- To every line associate momentum $k_{j}$. Momentum of external lines satisfy $-k_{i}^{2}=\Delta_{i}$. Momentum conservation must hold for the whole amplitude, and at every vertex.

- To every internal line corresponding to a scalar of conformal dimension $\delta_{k}$, assign an integer $n_{k}$ and a propagator:

$$
\frac{1}{2 n_{j} ! \Gamma\left(1+\delta_{j}+n_{j}-h\right)} \frac{-1}{+k_{j}^{2}+\left(\delta_{j}+2 n_{j}\right)}
$$

- In $g^{(m)} \phi^{m}$ theory, the vertex connecting lines with dimension $\Delta_{i}$, integers $n_{i}$, is given by

$$
\begin{aligned}
V_{\left[n_{1}, \ldots, n_{m}\right]}^{\Delta_{1} \ldots \Delta_{m}}= & g^{(m)} \Gamma\left(\frac{\sum_{i} \Delta_{i}-2 h}{2}\right)\left(\prod_{i=1}^{n}\left(1-h+\Delta_{i}\right)_{n_{i}}\right) \\
& F_{A}^{(m)}\left(\frac{\sum_{i=1}^{n} \Delta_{i}-2 h}{2},\left\{-n_{1}, \ldots,-n_{m}\right\},\left\{1+\Delta_{1}-h, \ldots, 1+\Delta_{m}-h\right\} ; 1, \ldots, 1\right)
\end{aligned}
$$

where $(a)_{m}$ is the Pochhammer symbol and $F_{A}^{(m)}$ is the Lauricella function of $m$ variables ${ }^{3}$

- The Mellin amplitude is obtained by summing over all non-zero integer $n_{i}$.

If this conjecture is correct, then correlation functions in the purely scalar sector are completely solved at tree level (other kinds of interactions, such as those including derivatives, can be easily included [21]). A proof of these rules will require a better understanding of how lower-point Mellin amplitudes are combined into higher point ones.

An important result in this work, is a simplified formalism for the calculation of correlation functions of objects with indices, such as currents and stress-tensors. We shall find that the bulk to boundary propagators of these objects can be written as certain differential operators $D^{M A}$ acting on scalar propagators. For instance the three-point current Mellin amplitude $M_{3}^{M_{1} M_{2} M_{3}}$ may be written schematically as

$$
M_{3}^{M_{1} M_{2} M_{3}}=D^{M_{1} A_{1}} D^{M_{2} A_{2}} D^{M_{3} A_{3}} \tilde{M}_{A_{1} A_{2} A_{3}} .
$$

The $D$ operators act as projectors, taking the reduced Mellin amplitude $\tilde{M}$ onto a conformally invariant subspace. As such, the reduced Mellin amplitude $\tilde{M}$ is dramatically simpler then the full amplitude. In particular its tensorial structure is essentially the same one that would appear in a flat space scattering amplitude, upon certain identifications. This simplification holds for arbitrary $n$-point functions, of fields with arbitrary spin. In particular, in this paper we shall carry out as an example the calculation of a four-current Witten diagram involving current exchange in Yang-Mills theory. With some more work, the fourpoint function of the stress-tensor should be obtainable, since the difficulties involved are essentially the same that are involved in a flat space scattering calculation.

\footnotetext{
${ }^{3}$ The definition is given in equation (4.23). Also, see references [26-28].
} 
The usage of the embedding formalism also clarifies the requirements of conformal invariance. Consider for instance the current three-point amplitude,

$$
\left\langle J^{M_{1}}\left(P_{1}\right) J^{M_{2}}\left(P_{2}\right) J^{M_{3}}\left(P_{3}\right)\right\rangle
$$

where all $P_{i}^{M_{i}}$ are $d+2$ dimensional vectors which square to zero. To get the $d$-dimensional amplitude we must pull back the $M_{i}$ indices to $\mu$ indices in $d$ dimensions. This only makes sense if the $M_{i}$ indices are transverse [24], that is, if:

$$
P_{i, M_{i}}\left\langle J^{M_{1}}\left(P_{1}\right) J^{M_{2}}\left(P_{2}\right) J^{M_{3}}\left(P_{3}\right)\right\rangle=0
$$

for any $i$. This requirement strongly constrains the form of the amplitude. There are essentially two building blocks

$$
\begin{aligned}
X_{i j}^{M_{k}} & =\left(\frac{P_{i}^{M_{k}}}{P_{i} \cdot P_{k}}-\frac{P_{j}^{M_{k}}}{P_{j} \cdot P_{k}}\right) \\
I^{M_{i} M_{j}} & =\eta^{M_{i} M_{j}}-\frac{P_{i}^{M_{j}} P_{j}^{M_{i}}}{P_{i} \cdot P_{j}}
\end{aligned}
$$

which satisfy $P_{M_{k}} X_{i j}^{M_{k}}=P_{M_{i}} I^{M_{i} M_{j}}=P_{M_{j}} I^{M_{i} M_{j}}=0$. From these we can construct the tensorial structure of any conformally invariant amplitude. In our example, we find that the amplitude must take the form

$$
\left\langle J^{M_{1}}\left(P_{1}\right) J^{M_{2}}\left(P_{2}\right) J^{M_{3}}\left(P_{3}\right)\right\rangle \propto a X_{12}^{M_{3}} X_{13}^{M_{2}} X_{12}^{M_{1}}+b\left(X_{12}^{M_{3}} \frac{I^{M_{1} M_{2}}}{P_{1} \cdot P_{2}}+\text { perms }\right)
$$

which is correct [18]. However, the reasoning is more general, and it applies to any $n$-point amplitude of any integer spin field.

The layout of this paper is as follows. In the next section we set up our formalism, describing in detail the embedding formalism, and the form of the bulk-to-bulk and bulk-toboundary propagators that will be used throughout the paper. In section 3 we review some of the results of [21], computing the Mellin amplitude corresponding to a scalar four-point function in $\phi^{3}$ theory. This will serve as the starting point and motivation for computing higher point amplitudes, in the quest to understand whether Mellin amplitudes can be described by a set of Feynman rules. In sections 4 and 5 we compute five and six-point amplitudes respectively. The form of the amplitudes is consistent with the Feynman rules we described previously, and we read off the general cubic vertex involving three descendant fields, given in terms of the Lauricella function of three arguments. In section 7 we turn our attention to correlators of spin-1 fields. We start by reproducing in a much simpler fashion several computations which have appeared previously in the literature: namely correlators $\langle J \mathcal{O O}\rangle,\langle J J J\rangle$ and a current exchange diagram in scalar theory. Putting all the ingredients together we are able to explicitly compute a current 4-point function. We finish with a brief discussion of our results and prospects for future work.

Note Added. While this work was being completed, we became aware of the work of [29] which partially overlap with some of our results. We thank the authors for granting us access to an early version of their manuscript. 


\section{Preliminaries}

\subsection{Embedding formalism}

Throughout this paper we shall make strong use of the embedding formalism. In this formalism, $A d S_{d+1}$ space is seen as a curved surface embedded in flat Minkowski space $M_{d+2}$. The Minkowski space metric is denoted $\eta^{M N}$, and it is written as

$$
d s^{2}=-\mathrm{d} X^{+} \mathrm{d} X^{-}+\delta_{m n} \mathrm{~d} X^{m} \mathrm{~d} X^{n} .
$$

That is, we describe the first two directions with lightcone coordinates. AdS coordinate vectors $X^{M}$ satisfy $X \cdot X=-R^{2}$ whereas AdS boundary coordinates $P^{M}$ are defined by $P^{2}=0$. We are also free to perform rescalings $P \rightarrow \alpha P, \alpha>0$, and as such amplitudes $M\left(P_{i}\right)$ satisfying conformal invariance should also scale: $M\left(P_{i}\right) \rightarrow \alpha^{\Delta} M$ for some $\Delta$. To fix notation we choose

- $P_{i}$ - fixed boundary points.

- $Q_{i}$ - boundary points integrated over.

- $X_{i}$ - AdS bulk coordinate.

We will also set throughout the rest of this paper the AdS radius to one. Dependence on this quantity can be recovered by dimensional analysis. Useful parameterizations of AdS and its boundary are

$$
X^{A}\left(x^{a}\right)=\frac{1}{x_{0}}\left(1, x_{0}^{2}+x^{2}, x^{\mu}\right), \quad P^{M}\left(x^{\mu}\right)=\left(1, y^{2}, y^{\mu}\right) .
$$

where $x^{\mu}$ is a $d$-dimensional vector and $x^{2}=x^{\mu} x_{\mu}$. In this way we have for instance:

$$
\begin{aligned}
P_{i j} & \equiv-2 P_{i} \cdot P_{j}=\left(y_{i}-y_{j}\right)^{2} \\
-2 P \cdot X & =\frac{1}{x_{0}}\left(x_{0}^{2}+(x-y)^{2}\right) .
\end{aligned}
$$

Objects with indices $T_{A_{1} \ldots}$ are tensors in AdS if they satisfy $X^{A_{1}} T_{A_{1} \ldots}=0[24,30]$. To implement this transversality condition one may use the projector

$$
U^{A B}=\eta^{A B}+X^{A} X^{B} .
$$

It is also useful to know how to write such $d+2$ tensors in terms of $d$-dimensional ones. In other words, we need to be able to pull-back $M$ indices to $\mu$ indices, and this is achieved by use of the objects

$$
\zeta_{\mu}^{M}(P)=\frac{\partial P^{M}\left(y^{\mu}\right)}{\partial y^{\mu}}, \quad \varphi_{a}^{M}(X)=\frac{\partial X^{M}\left(x^{\mu}\right)}{\partial x^{a}} .
$$

Because of the constraints $X^{2}=-1, P^{2}=0$, we necessarily have $\zeta_{\mu}(P) \cdot P=\varphi_{a}(X) \cdot X=0$. Using the parameterization of AdS and its boundary given in (2.2), we find the following 




Figure 1. A Witten diagram involving scalar fields.

useful identities:

$$
\begin{aligned}
\zeta_{\mu}(y) \cdot P\left(y^{\prime}\right) & =y_{\mu}^{\prime}-y_{\mu}, \\
\zeta_{\mu}(y) \cdot X(x) & =\frac{1}{x_{0}}\left(x_{\mu}-y_{\mu}\right), \\
\varphi_{0}(x) \cdot P(y) & =\frac{1}{2} \frac{(y-x)^{2}-x_{0}^{2}}{x_{0}^{2}} \\
\varphi_{\mu}(x) \cdot P(y) & =\frac{1}{x_{0}}\left(y_{\mu}-x_{\mu}\right) \\
\zeta_{\mu}(x) \cdot \zeta_{\nu}(y) & =\eta_{\mu \nu} \\
\varphi_{a}(x) \cdot \varphi_{b}(x) & =g_{a b} . \\
\varphi_{\mu}(x) \cdot \zeta_{\nu}(y) & =\frac{1}{x_{0}} \eta_{\mu \nu}
\end{aligned}
$$

In the following we will label indices that are to be contracted with $\zeta_{\mu}^{M}$ as $M, N, P, \ldots$, whereas "AdS" indices will be labelled $A, B, C \ldots$ This provides a practical distinction between boundary and bulk indices, although in the embedding formalism no such distinction exists.

\subsection{Boundary-bulk propagators}

In the AdS/CFT correspondence, conformal correlation functions can be calculated via Witten diagrams [1]. A typical diagram is shown in figure 1.

Such a diagram is made up of three ingredients, namely external lines which connect to the boundary of AdS, internal lines, and vertices. The vertices are simple to write down and are easy to read off from the gravitational lagrangian. External lines are bulkto-boundary propagators, propagating some field perturbation inserted on the boundary into the bulk, and internal lines are bulk-to-bulk propagators. To compute the amplitude we write down a propagator for each line, and integrate over all possible positions of the interaction vertices. In the following we shall give expressions for these propagators in the embedding formalism. Consider first the case where the perturbation corresponds to a 
scalar operator of conformal dimension $\Delta_{i}$. Then the propagator can be written as

$$
E_{i}(P, X)=\frac{C_{i}}{(-2 P \cdot X)^{\Delta_{i}}}=\frac{1}{2 \pi^{h} \Gamma\left(1+\Delta_{i}-h\right)} \int_{0}^{+\infty} \frac{\mathrm{d} t_{i}}{t_{i}} t_{i}^{\Delta_{i}} e^{2 t_{i} P \cdot X} .
$$

Here $i$ is shorthand notation for denoting the field in question and its conformal dimension, and the constants are

$$
C_{i}=\frac{\Gamma\left(\Delta_{i}\right)}{2 \pi^{h} \Gamma\left(1+\Delta_{i}-h\right)}, \quad h \equiv d / 2 .
$$

It is easy to check using our expression (2.4) that this reduces to the usual bulk-to-boundary propagator

$$
E_{i}(P, X) \simeq\left(\frac{x_{0}}{x_{0}^{2}+(y-x)^{2}}\right)^{\Delta_{i}} .
$$

However, the most convenient expression to use is the Schwinger parameterized form appearing on the right of (2.8), and this will be the one we will be using throughout this paper.

Now consider the bulk-to-boundary propagator of a spin-1 field. Such a propagator takes the form: ${ }^{4}$

$$
E_{i}^{M A}(P, X)=\frac{1}{2 \pi^{h} \Gamma\left(1+\Delta_{i}-h\right)} \int_{0}^{+\infty} \frac{\mathrm{d} t_{i}}{t_{i}} t_{i}^{\Delta_{i}} J^{M A} e^{2 t_{i} P \cdot X} .
$$

That is, it is given by the product of some tensor structure, to propagate indices, and the scalar propagator of a field of dimension $\Delta_{i}$. For a Yang-Mills field we will have $\Delta_{i}=d-1$, but we shall keep it arbitrary for now. Requiring transversality of the tensor structure both in AdS and at its boundary fixes $J^{M A}$ :

$$
P_{M} J^{M A}=J^{M A} X_{A}=0 \quad \Rightarrow \quad J^{M A}=\eta^{M A}-\frac{P^{A} X^{M}}{P \cdot X}
$$

The tensor $J^{M A}$ is a projector, as may be easily checked. It serves the two-fold purpose of making transverse in $X$ objects which contract it on the right, and transverse in $P$ objects which contract it on the left. The reader may check that the propagator written above reduces to the right one for a spin-one field upon the use of the identities (2.7). In fact, using $J^{M A}$, we can write down the bulk-to-boundary propagator for a field of any spin - we just multiply several $J^{M A}$ together and symmetrize appropriately its indices to get the right representation. In particular we can do this to obtain the bulk-to-boundary propagator of the graviton. Before we do this however, we notice that there is an alternative representation of the propagator which will be very useful by using the identity:

$$
\begin{aligned}
\int \frac{\mathrm{d} t}{t} t^{\Delta} \frac{P^{A} X^{M}}{P \cdot X} e^{2 t P \cdot X} & =\int \frac{\mathrm{d} t}{t} t^{\Delta} \frac{(-2 t)}{\Delta} P^{A} X^{M} e^{2 t P \cdot X} \\
& =-\int \frac{\mathrm{d} t}{t} t^{\Delta} \frac{P^{A}}{\Delta} \frac{\partial}{\partial P^{M}} e^{2 t P \cdot X},
\end{aligned}
$$

we can write $E_{i}^{M A}(P, X)=D_{\Delta}^{M A} E_{i}(P, X)$ with the operator

$$
D_{\Delta}^{M A} \equiv \eta^{M A}+\frac{1}{\Delta} P^{A} \frac{\partial}{\partial P^{M}} \equiv \eta^{M A}+\frac{1}{\Delta} P^{A} \partial_{M_{1}} .
$$

\footnotetext{
${ }^{4}$ Our normalization differs from that of [7] by a factor of $d-1$.
} 
Similarly, for the spin-2 case, we can also write the bulk-to-boundary propagator in terms of an operator acting on the scalar propagator:

$$
\begin{aligned}
E_{i}^{M_{1} M_{2} A B}(P, X) & =D_{2, \Delta}^{M_{1} M_{2} A B} E_{i}(P, X), \\
D_{2, \Delta}^{M_{1} M_{2} A_{1} A_{2}} & =\eta^{M_{1} A_{1}} \eta^{M_{2} A_{2}}+\frac{1}{\Delta}\left(\eta^{M_{1} A_{1}} P^{A_{2}} \partial_{M_{2}}+1 \leftrightarrow 2\right)+\frac{P^{A_{1}} P^{A_{2}}}{\Delta(\Delta+1)} \partial_{M_{1}} \partial_{M_{2}}
\end{aligned}
$$

Once again, in applications we should take $\Delta=d$ in the above.

\section{$2.3 \quad$ Bulk-to-bulk propagators}

Next we consider the bulk-to-bulk propagators. These are associated with internal lines in Witten diagrams. For ease of notation, we will henceforth denote the conformal dimension of fields propagating in these internal lines by a lower case $\delta$, and dimensions of fields on external lines by a capital $\Delta$. Then, for a scalar field of dimension $\delta$, the bulk-to-bulk propagator $G_{B B}(X, Y)$ can be written in the embedding formalism as

$$
G_{B B}\left(X_{1}, X_{2}\right)=\int_{-i \infty}^{+\infty} \frac{\mathrm{d} c}{2 \pi i} f_{\delta, 0}(c) \int_{\partial \mathrm{AdS}} \mathrm{d} Q \int \widetilde{d^{2} s_{c}} e^{2 s Q \cdot X+2 \bar{s} Q \cdot Y}
$$

with

$$
f_{\delta, 0}(c) \equiv \frac{1}{2 \pi^{2 h}\left[(\delta-h)^{2}-c^{2}\right]} \frac{1}{\Gamma(c) \Gamma(-c)}, \quad \widetilde{\mathrm{d}^{2} s_{c}} \equiv \frac{\mathrm{d} s}{s} \frac{\mathrm{d} \bar{s}}{\bar{s}} s^{h+c} \bar{s}^{h-c}
$$

It is remarkable that this can be seen as the product of two boundary to bulk propagators of states with unphysical conformal dimensions $h \pm c$, glued together by the integration over the boundary point $Q$ and over $c$. Bulk to bulk propagators of fields with spin will have the same structure as we shall see shortly. The fact that the dependence of the propagator on $X$ and $Y$ factorises simplifies calculations a great deal, since then an $n$-point amplitude can be obtained by appropriately gluing lower-point amplitudes. In particular, this allows one to ultimately reduce an $n$-point amplitudes to a gluing of three point amplitudes, analogously to (but not quite) BCFW [31] recursion relations.

The bulk-to-bulk propagator for a spin-one field is written in a similar fashion to the spin-zero case [32]:

$$
\begin{aligned}
& G_{B B}^{A B}\left(X_{1}, X_{2}\right)= \\
& \quad \int_{-i \infty}^{+\infty} \frac{\mathrm{d} c}{2 \pi i} f_{\delta, 1}(c) \int_{\partial \mathrm{AdS}} \mathrm{d} Q \int \frac{\mathrm{d} s}{s} s^{h+c}\left(D_{h+c}^{M A} e^{2 s Q \cdot X_{1}}\right) \eta_{M N} \int \frac{\mathrm{d} \bar{s}}{\bar{s}} \bar{s}^{h-c}\left(D_{h-c}^{N B} e^{2 \bar{s} Q \cdot X_{2}}\right)
\end{aligned}
$$

with

$$
f_{\delta, 1}=f_{\delta, 0} \frac{h^{2}-c^{2}}{(\delta-h)^{2}-c^{2}}, \quad \delta=d-1
$$

and $D_{\Delta}^{M A}$ the operator defined previously in (2.15). Finally, the bulk-to-bulk graviton propagator can be obtained by the replacements [32]

$$
\begin{aligned}
f_{\delta, 1} \rightarrow f_{\delta, 2} & =f_{\delta, 0}\left[(h+1)^{2}-c^{2}\right] \\
D^{M A} & \rightarrow D^{M_{1} M_{2} A_{1} A_{2}} \\
\eta_{M N} & \rightarrow \mathcal{E}_{M_{1} M_{2}, N_{1} N_{2}}
\end{aligned}
$$




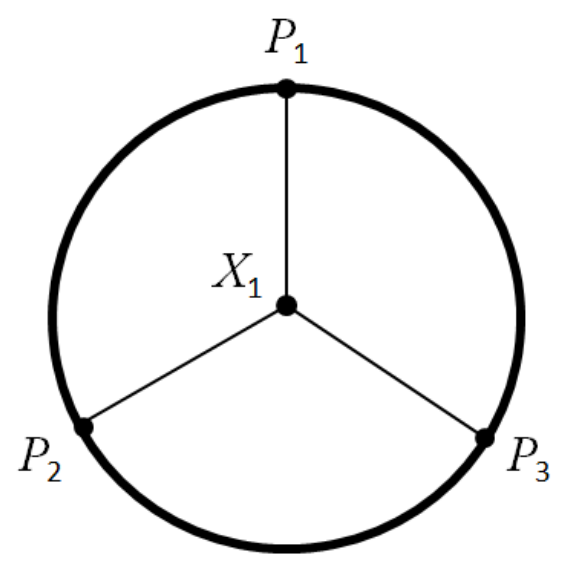

Figure 2. Scalar three-point function.

with $\mathcal{E}$ given by

$$
\mathcal{E}_{M_{1} M_{2} N_{1} N_{2}} \equiv \frac{1}{2}\left(\eta_{M_{1} N_{1}} \eta_{M_{2} N_{2}}+\eta_{M_{1} N_{2}} \eta_{M_{2} N_{1}}\right)-\frac{1}{d} \eta_{M_{1} M_{2}} \eta_{N_{1} N_{2}} .
$$

The appearance of $d$ instead of $d+2$ in the above will be explained in section (5). For now it is sufficient to notice that in order to get the correct $d$ dimensional index structure we must have $\mathcal{E}$ of this form.

\section{Warm-up: 3 and 4-point scalar correlation functions}

\subsection{3 -point vertex}

Now that we have expressions for all the propagators, we are ready to compute some amplitudes. We will see that using both the embedding formalism and the Schwinger parameterized form of the propagators naturally leads to the appearance of the Mellin transform of the amplitudes, as well as simplifying considerably the calculations.

As a warm-up, consider first a simple theory of massive scalars in $A d S_{d+1}$ interacting via a cubic potential:

$$
S_{\phi}=\int \mathrm{d}^{d+1} x \sqrt{g}\left(\sum_{i} \frac{1}{2}\left(\partial \phi_{i}\right)^{2}+\frac{1}{2} m_{i}^{2} \phi_{i}^{2}+\frac{g}{3 !}\left(\sum_{i} \phi_{i}\right)^{3}\right) .
$$

The conformal dimension of the operator $\mathcal{O}_{i}$ dual to $\phi_{i}$ is then $\Delta_{i}=h \pm \sqrt{h^{2}+m_{i}^{2}}$. We start by calculating a scalar three point function, described by the Witten diagram of figure 2 . To each leg connected to the boundary we associate a boundary to bulk propagator $E_{i}$. We are then instructed to integrate over the interaction point in the bulk of AdS, so that the overall amplitude is given by

$$
\begin{aligned}
A(1,2,3) & \equiv\left\langle\mathcal{O}_{1}\left(P_{1}\right) \mathcal{O}_{2}\left(P_{2}\right) \mathcal{O}_{3}\left(P_{3}\right)\right\rangle=g \int_{\mathrm{AdS}} \mathrm{d} X E_{1}\left(P_{1}, X\right) E_{2}\left(P_{2}, X\right) E_{3}\left(P_{3}, X\right), \\
& =g \mathcal{E}_{3} \int_{0}^{+\infty} \prod_{i=1}^{3} \frac{\mathrm{d} t_{i}}{t_{i}} t_{i}^{\Delta_{i}} \int_{\mathrm{AdS}} \mathrm{d} X \exp \left(2\left(t_{1} P_{1}+t_{2} P_{2}+t_{3} P_{3}\right) \cdot X\right)
\end{aligned}
$$


with $\mathcal{E}_{3}=\prod_{i=1}^{3} \frac{C_{i}}{\Gamma\left(\Delta_{i}\right)}$. To proceed we use the result (A.1), whereupon we obtain

$$
A(1,2,3)=g \pi^{h} \mathcal{E}_{3} \Gamma\left(\frac{\sum_{i}^{n} \Delta_{i}-2 h}{2}\right) \int \prod_{i=1}^{3} \frac{\mathrm{d} t_{i}}{t_{i}} t_{i}^{\Delta_{i}} \exp \left(-t_{1} t_{2} P_{12}-t_{1} t_{3} P_{13}-t_{2} t_{3} P_{23}\right) .
$$

with $P_{i j} \equiv-2 P_{i} \cdot P_{j}$. The integrals may be directly performed by doing a change of variables,

$$
t_{1}=\sqrt{\frac{m_{3} m_{2}}{m_{1}}}, \quad t_{2}=\sqrt{\frac{m_{3} m_{1}}{m_{2}}}, \quad t_{3}=\sqrt{\frac{m_{1} m_{2}}{m_{3}}} .
$$

obtaining

$$
A(1,2,3)=\frac{\pi^{h}}{2} g \Gamma\left(\frac{\sum_{i}^{3} \Delta_{i}-2 h}{2}\right) \mathcal{E}_{3} \prod_{i=1}^{3} \int \frac{\mathrm{d} m_{i}}{m_{i}} m_{i}^{\delta_{j k}} e^{-m_{i} P_{j k}}
$$

where it should be understood that if $i=1, j k=23$, etc, and

$$
\delta_{12}=\frac{\Delta_{1}+\Delta_{2}-\Delta_{3}}{2}, \quad \delta_{23}=\frac{\Delta_{2}+\Delta_{3}-\Delta_{1}}{2}, \quad \delta_{13}=\frac{\Delta_{1}+\Delta_{3}-\Delta_{2}}{2} .
$$

The integrations are now trivial and one obtains

$$
A(1,2,3)=\frac{\pi^{h}}{2} g \Gamma\left(\frac{\sum_{i}^{3} \Delta_{i}-2 h}{2}\right) \mathcal{E}_{3} \prod_{i<j}^{3} \Gamma\left(\delta_{i j}\right)\left(P_{i j}\right)^{-\delta_{i j}}
$$

In general, we define the normalization constant in (1.6) by

$$
\mathcal{N} \equiv \frac{\pi^{h}}{2} \prod_{i=1}^{n} \frac{C_{i}}{\Gamma\left(\Delta_{i}\right)}
$$

In this particular case, this gives for the three-point Mellin amplitude:

$$
M_{3}=g \Gamma\left(\frac{\sum_{i}^{3} \Delta_{i}-2 h}{2}\right) \equiv V_{[0,0,0]}^{\Delta_{1}, \Delta_{2}, \Delta_{3}}
$$

The notation for the vertex $V$ will become clear later on. For the practical purpose of computing the Mellin amplitude, we need not worry about the overall normalization constant $\mathcal{N}$, since to restore it, one can simply include a factor $C_{i} / \Gamma\left(\Delta_{i}\right)$ for each external leg. As such we will for the most part omit it from our calculations.

\subsection{4-point exchange diagram}

Now let us tackle an example where there is an intermediate state being exchanged in the bulk. We consider a four point amplitude of operators $\mathcal{O}_{i}$ and dimension $\Delta_{i}, i=1, \ldots, 4$, where a scalar of conformal dimension $\delta$ is being exchanged in the "s-channel". The Witten diagram is shown in figure 3 . Let us denote the corresponding amplitude by $I_{s}$. There are now two three point interactions happenning at points $X_{1}, X_{2}$, over which we must integrate over. The amplitude is written

$$
I_{s}=g^{2} \int_{\mathrm{AdS}} \mathrm{d} X_{1} \int_{\mathrm{AdS}} \mathrm{d} X_{2} E_{1}\left(P_{1}, X_{1}\right) E_{2}\left(P_{2}, X_{1}\right) G_{B B}\left(X_{1}, X_{2}\right) E_{3}\left(P_{3}, X_{2}\right) E_{4}\left(P_{4}, X_{2}\right) .
$$




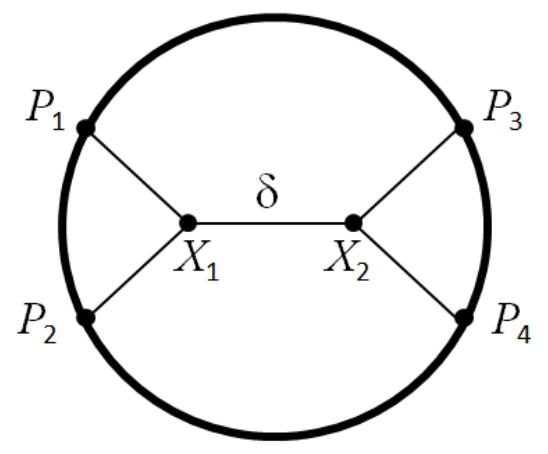

Figure 3. Scalar exchange diagram.
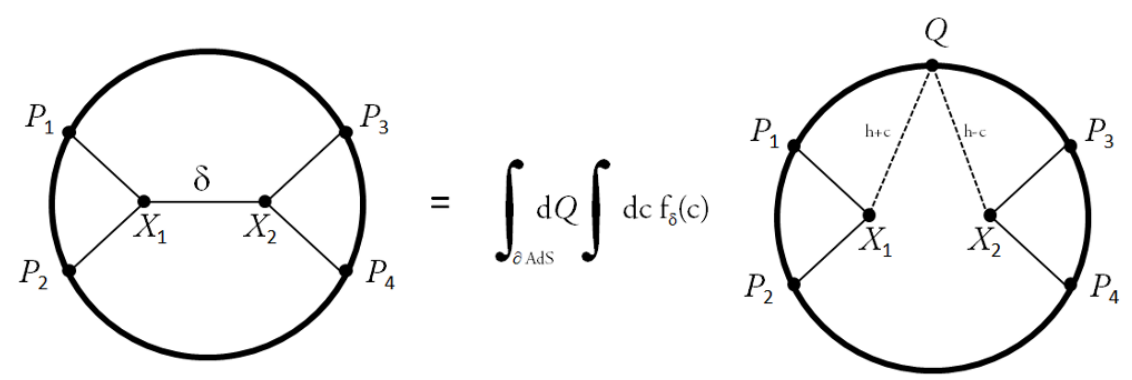

Figure 4. Four-point amplitudes result from gluing a pair of three-point amplitudes.

As we've seen in section 2.3 the dependence of the bulk-to-bulk propagator on $X_{1}, X_{2}$ factorises, and the amplitude becomes

$$
I_{s}=\int_{-i \infty}^{+i \infty} \frac{\mathrm{d} c}{2 \pi i} f_{\delta}(c) \int_{\partial \mathrm{AdS}} \mathrm{d} Q A\left(P_{1}, P_{2}, Q_{+}\right) A\left(Q_{-}, P_{3}, P_{4}\right) .
$$

with

$$
\begin{aligned}
& A\left(P_{1}, P_{2}, Q_{+}\right)=g \int_{0}^{+\infty} \frac{\mathrm{d} t_{1}}{t_{1}} \frac{\mathrm{d} t_{2}}{t_{2}} \frac{\mathrm{d} s}{s} t_{1}^{\Delta_{1}} t_{2}^{\Delta_{2}} s^{h+c} \int_{\text {AdS }} \mathrm{d} X_{1} e^{2\left(t_{1} P_{1}+t_{2} P_{2}+s Q\right) \cdot X_{1}}, \\
& A\left(P_{3}, P_{4}, Q_{-}\right)=g \int_{0}^{+\infty} \frac{\mathrm{d} t_{3}}{t_{3}} \frac{\mathrm{d} t_{4}}{t_{4}} \frac{\mathrm{d} \bar{s}}{\bar{s}} t_{3}^{\Delta_{3}} t_{4}^{\Delta_{4}} s^{h-c} \int_{\text {AdS }} \mathrm{d} X_{2} e^{2\left(t_{3} P_{3}+t_{4} P_{4}+\bar{s} Q\right) \cdot X_{2}} .
\end{aligned}
$$

These are simply three-point amplitudes, which we have already computed. This decomposition is shown diagramatically in figure 4 .

Since the bulk-to-bulk propagators always factorise in this way, any $n$-point amplitude will be the result of gluing together several three point amplitudes. We need a useful notation for denoting these, as they will occur often. We choose:

$$
A_{\Delta_{i}, \Delta_{j}, h \pm c_{k}}\left(P_{i}, P_{j}, Q_{i}\right) \equiv A\left(i, j, c_{k}^{ \pm}\right) .
$$

In case a given three point amplitude contains two $Q^{\prime} s$ then it will also depend on two $c$ parameters. To every boundary coordinate integration there will correspond a single $c$, so that the above notation is consistent. 
To compute integrals such as the one in (3.11), the standard procedure is to introduce Schwinger parameters to exponentiate the powers of $P_{i j}$. These are the $t$ and $s$ parameters appearing in the expressions for the propagators. In practice, we always start by first performing the $X$ integrations so that we are left with expressions of the form:

$$
A\left(i, j, c^{ \pm}\right)=g_{i, j, c^{ \pm}} \int_{0}^{+\infty} \frac{\mathrm{d} t_{1}}{t_{1}} \frac{\mathrm{d} t_{2}}{t_{2}} \frac{\mathrm{d} s}{s} t_{1}^{\Delta_{1}} t_{2}^{\Delta_{2}} s^{h+c} \exp \left[-t_{1} t_{2} P_{12}+2 s\left(t_{1} P_{1}+t_{2} P_{2}\right) \cdot Q\right]
$$

with

$$
g_{i, j, c^{ \pm}} \equiv g \pi^{h} \Gamma\left(\frac{\Delta_{i}+\Delta_{j}+(h \pm c)-2 h}{2}\right) .
$$

In the particular case at hand, if we write both 3-point amplitudes in this fashion it is easy to see that the $Q$ integral which must be performed is precisely of the form (A.5). We then get

$$
\begin{aligned}
A_{4}= & g^{2}\left(2 \pi^{3 h}\right) \int_{-i \infty}^{+i \infty} \frac{\mathrm{d} c}{2 \pi i} f_{\delta, 0}(c) \int \widetilde{\mathrm{d}^{2} s} \Gamma\left(\frac{\Delta_{1}+\Delta_{2}+c-h}{2}\right) \Gamma\left(\frac{\Delta_{3}+\Delta_{4}-c-h}{2}\right) \\
& \int \prod_{i=1}^{4} \frac{\mathrm{d} t_{i}}{t_{i}} t_{i}^{\Delta_{i}} \exp \left[-\left(1+s^{2}\right) t_{1} t_{2} P_{12}-\left(1+\bar{s}^{2}\right) t_{1} t_{2} P_{34}-s \bar{s} \sum_{(i j)}^{\prime} t_{i} t_{j} P_{i j}\right] .
\end{aligned}
$$

where the primed sum indicates we are summing over the "cross-links" 13, 14, 23, 24. We can now use Symanzik's star formula (which we review in appendix B), to show that the amplitude $I_{s}$ can be written in the form (1.6), with a Mellin amplitude given by

$$
M\left(\delta_{i j}\right)=2 \int_{-i \infty}^{+i \infty} \frac{\mathrm{d} c}{2 \pi i} f_{\delta, 0}(c) I(12, h, c) I(34, h,-c),
$$

with e.g.

$$
I(12, h, c)=g_{1,2, c^{+}} \int_{0}^{+\infty} \frac{\mathrm{d} s}{s} s^{h+c-\sum^{\prime} \delta_{i j}}\left(1+s^{2}\right)^{-\delta_{12}},
$$

The integrals can be evaluated in terms of gamma functions. Using the relations (1.9) to express the $\delta_{i j}$ parameters in terms of Mandelstam invariants we find

$$
M\left(s_{12}\right)=\frac{g^{2}}{\Gamma\left(\frac{\Delta_{1}+\Delta_{2}-s_{12}}{2}\right) \Gamma\left(\frac{\Delta_{3}+\Delta_{4}-s_{12}}{2}\right)} \int_{-i \infty}^{+i \infty} \frac{\mathrm{d} c}{2 \pi i} \frac{l_{h}(c) l_{h}(-c)}{(\delta-h)^{2}-c^{2}}
$$

where we have defined

$$
l_{h}(c)=\frac{\Gamma\left(\frac{h+c-s_{12}}{2}\right) \Gamma\left(\frac{\Delta_{1}+\Delta_{2}+c-h}{2}\right) \Gamma\left(\frac{\Delta_{3}+\Delta_{4}+c-h}{2}\right)}{2 \Gamma(c)} .
$$

The Mellin-Barnes integral can be exactly evaluated in terms of a hypergeometric ${ }_{3} F_{2}$ function [21]:

$$
\begin{aligned}
M\left(s_{12}\right)= & \frac{1}{2} \frac{g^{2}}{s_{12}-\delta} \frac{\Gamma\left(\frac{\Delta_{1}+\Delta_{2}+\delta-h}{2}\right) \Gamma\left(\frac{\Delta_{3}+\Delta_{4}+\delta-h}{2}\right)}{\Gamma(1+\delta-h)} \\
& { }_{3} F_{2}\left(\frac{2-\Delta_{1}-\Delta_{2}+\delta}{2}, \frac{2-\Delta_{3}-\Delta_{4}+\delta}{2}, \frac{\delta-s_{12}}{2} ; \frac{2+\delta-s_{12}}{2}, 1+\delta-h ; 1\right) .
\end{aligned}
$$


It is more useful for us however, to write the amplitude in a different fashion. Since the integral must lead to a meromorphic of $s_{12}$, we can write the result as a Laurent series in $s_{12}$. The poles of this function are found by examining when the $c$ integration contour gets pinched between two poles of the integrand. We can choose the contour such that this happens when $c=\delta-h$ and $s_{12}=\delta+2 n$, with $n$ a positive integer. Then it is easy to find

$$
M\left(s_{12}\right)=\sum_{n=0}^{+\infty} \frac{P_{n}^{\delta}}{s_{12}-\delta-2 n} V_{[0,0, n]}^{\Delta_{1}, \Delta_{2}, \delta} V_{[0,0, n]}^{\Delta_{3}, \Delta_{4}, \delta}+\ldots
$$

The dots represent polynomial contributions to the amplitude, but as it happens, in this particular case they are vanishing, as can be checked by computing the amplitude exactly, and the sum of poles is therefore the full amplitude. We have defined the vertices and propagator normalization,

$$
\begin{aligned}
V_{[0,0,0]}^{\Delta_{1}, \Delta_{2}, \Delta_{3}} & =g \Gamma\left(\frac{\sum_{i}^{3} \Delta_{i}-2 h}{2}\right) \\
V_{\left[0,0, n_{1}\right]}^{\Delta_{1}, \Delta_{2}, \Delta_{3}} & =V_{[0,0,0]}^{\Delta_{1}, \Delta_{2}, \Delta_{3}}\left(1-\frac{1}{2} \sum_{i}^{3} \Delta_{i}+\Delta_{3}\right)_{n_{1}} \\
P_{n}^{\delta} & =[2 n ! \Gamma(1+\delta-h+n)]^{-1}
\end{aligned}
$$

with the help of the Pochhammer symbol $(a)_{m}=\Gamma(a+m) / \Gamma(a)$. The interpretation of this expression is clear: the Mellin amplitude is an infinite sum of products of three point vertices and a propagator. The sum runs over the propagating fields, which include a field with conformal dimension $\delta$ and its "descendants", with dimension $\delta+2 n$. From the above one reads off the three point Mellin amplitude of two fields of dimensions $\Delta_{1}, \Delta_{2}$, and one such descendant to be simply $V_{[0,0, n]}^{\Delta_{3}, \Delta_{4}, \delta}$. In particular for $n=0$ this reduces to the three point Mellin amplitude we previously computed.

This result suggests a set of Feynman rules for Mellin amplitudes, where to each internal line in a Witten diagram one associates an infinite sum of propagating fields (one primary and an infinite set of descendants), to each vertex one associates a factor $V_{[m, n, p]}^{\Delta_{1}, \Delta_{2}, \Delta_{3}}$, and for each line a normalization factor which is the inverse of $\Gamma\left(1+\Delta_{i}+n-h\right)$. These are of course nothing but the Feynman rules we conjectured in the introduction section. However, right now we do not yet know the form of the general vertex, which can involve up to three "descendants". In principle its form is directly fixed by kinematic considerations alone, that is, by conformal symmetry. In practice, to proceed we shall extract this vertex by evaluating higher point amplitudes. This provides a simple way of reading off the vertex, and will also act as a cross-check on our proposed Feynman rules.

Firstly we consider a five point amplitude. In such a diagram there is a vertex connecting two internal lines, and from it we will be able to read off $V_{[0, n, p]}^{\Delta_{1}, \Delta_{2}, \Delta_{3}}$. We will also explicitly see that these Feynman rules still work there. Finally, the full vertex may be obtained by considering a 6 -point amplitude. We shall see how the latter can be written as a product of three propagators and associated vertices, and read off $V_{[m, n, p]}^{\Delta_{1}, \Delta_{2}, \Delta_{3}}$. 


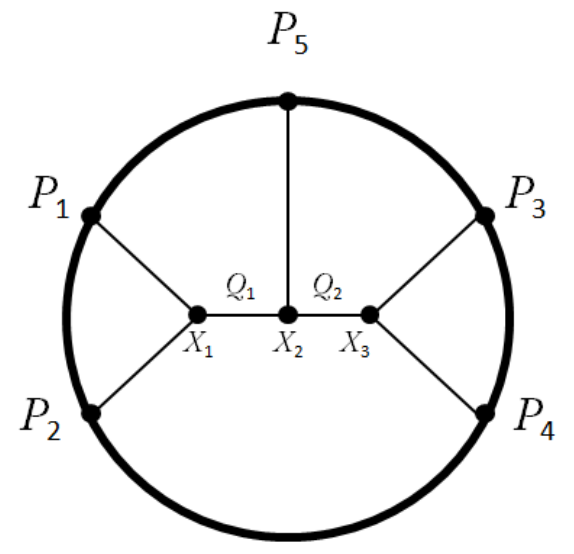

Figure 5. A five-point Witten diagram in scalar theory.

\section{Scalar higher-point amplitudes}

\section{$4.1 \quad 5$-point amplitude}

Consider the Witten diagram of figure 5, for a five point amplitude in cubic theory. The amplitude is given by ${ }^{5}$

$$
\begin{aligned}
A_{5}= & g^{3} \int \prod_{i}^{5} \frac{\mathrm{d} t_{i}}{t_{i}} t_{i}^{\Delta_{i}} \int_{-i \infty}^{+i \infty} \frac{\mathrm{d} c_{1} \mathrm{~d} c_{2}}{(2 \pi i)^{2}} f_{\delta_{1}}\left(c_{1}\right) f_{\delta_{2}}\left(c_{2}\right) \int \widetilde{\mathrm{d}^{2} s_{1}} \widetilde{\mathrm{d}^{2} s_{2}} \\
& \int_{\partial \mathrm{AdS}} \mathrm{d} Q_{1} \mathrm{~d} Q_{2} \int_{\text {AdS }} \mathrm{d} X_{1} \mathrm{~d} X_{2} \mathrm{~d} X_{3} \exp \left[2 X_{1} \cdot\left(t_{1} P_{1}+t_{2} P_{2}+s_{1} Q_{1}\right)+\right. \\
& \left.2 X_{3} \cdot\left(t_{3} P_{3}+t_{4} P_{4}+s_{2} Q_{2}\right)+2 X_{2} \cdot\left(t_{5} P_{5}+\bar{s}_{1} Q_{1}+\bar{s}_{2} Q_{2}\right)\right] .
\end{aligned}
$$

This looks quite complicated as it stands. However, we see that as expected from our general arguments in the previous section, each $X_{i}$ only couples to three coordinates coming into a vertex, and so we can immediately write

$$
A_{5}=g^{3} \int_{-i \infty}^{+i \infty} \frac{\mathrm{d} c_{1} \mathrm{~d} c_{2}}{(2 \pi i)^{2}} f_{\delta_{1}}\left(c_{1}\right) f_{\delta_{2}}\left(c_{2}\right) \int_{\partial \mathrm{AdS}} \mathrm{d} Q_{1} \mathrm{~d} Q_{2} A\left(1,2, c_{1}^{+}\right) A\left(3,4, c_{2}^{+}\right) A\left(5, c_{1}^{-}, c_{2}^{-}\right)
$$

Replacing the three point amplitudes for their Schwinger-parameterized expressions, we have an integrand of the form

$$
\begin{aligned}
\simeq \exp \left[-t_{1} t_{2} P_{12}-t_{3} t_{4} P_{34}\right] \exp [ & 2 Q_{1} \cdot\left(s_{1} t_{1} P_{1}+s_{1} t_{2} P_{2}+\bar{s}_{1} t_{5} P_{5}\right)+ \\
& \left.2 Q_{2} \cdot\left(s_{2} t_{3} P_{3}+s_{2} t_{4} P_{4}+\bar{s}_{2} t_{5} P_{5}\right)+2 \bar{s}_{1} \bar{s}_{2} Q_{1} \cdot Q_{2}\right]
\end{aligned}
$$

We now perform the $Q$ integrals, first $Q_{1}$ and $Q_{2}$. Consequently the result appears to break the symmetry of the diagram, but this will be restored later. The result is that the

\footnotetext{
${ }^{5}$ For economy of space we omit the external line normalization factors $C_{i} / \Gamma\left(\Delta_{i}\right)$, which are removed anyway upon passage to Mellin space.
} 
integrand becomes the exponential of a polynomial quadratic in the $P_{i}$ 's of the form

$$
\simeq \exp \left[-\sum_{i<j} Q_{i j} t_{i} t_{j} P_{i j}\right]
$$

Using Symanzik's star formula we obtain the Mellin amplitude

$$
\begin{aligned}
M_{5}= & g^{3}\left(4 \pi^{h}\right) \int_{-i \infty}^{+i \infty} \frac{\mathrm{d} c_{1} \mathrm{~d} c_{2}}{(2 \pi i)^{2}} f_{\delta_{1}}\left(c_{1}\right) f_{\delta_{2}}\left(c_{2}\right) g_{1,2, c_{1}^{+}} g_{3,4, c_{2}^{+}} g_{5, c_{1}^{-}, c_{2}^{-}} \\
& \int \widetilde{\mathrm{d}^{2} s_{1}} \widetilde{\mathrm{d}^{2} s_{2}}\left(1+s_{1}^{2}+s_{1}^{2} \bar{s}_{1}^{2} \bar{s}_{2}^{2}\right)^{-\delta_{12}}\left(1+s_{2}^{2}\right)^{-\delta_{34}}\left(1+\bar{s}_{1}^{2}\right)^{-\delta_{35}-\delta_{45}} \times \\
& \left(1+\bar{s}_{2}^{2}+\bar{s}_{1}^{2} \bar{s}_{2}^{2}\right)^{-\delta_{15}-\delta_{25}}\left(s_{1} \bar{s}_{1}\right)^{-\delta_{15}-\delta_{25}-\delta_{13}-\delta_{14}-\delta_{23}-\delta_{24}}\left(s_{2} \bar{s}_{2}\right)^{-\delta_{35}-\delta_{45}-\delta_{13}-\delta_{14}-\delta_{23}-\delta_{24}} .
\end{aligned}
$$

To proceed we must compute the $s_{i}$ integrals. The integrals of $s_{1}, s_{2}$ are simply performed and result in more Gamma functions. Using the Mandelstam invariant representation of the $\delta_{i j}$, the amplitude becomes

$$
\begin{aligned}
M_{5}= & g^{3} \pi^{4 h} \int_{-i \infty}^{+i \infty} \frac{\mathrm{d} c_{1} \mathrm{~d} c_{2}}{(2 \pi i)^{2}} f_{\delta_{1}}\left(c_{1}\right) f_{\delta_{2}}\left(c_{2}\right) \frac{\Gamma\left(\frac{-s_{12}+c_{1}+h}{2}\right) \Gamma\left(\frac{-s_{34}+c_{2}+h}{2}\right)}{\Gamma\left(\frac{\Delta_{1}+\Delta_{2}-s_{12}}{2}\right) \Gamma\left(\frac{\Delta_{3}+\Delta_{4}-s_{34}}{2}\right)} \Gamma\left(\frac{\Delta_{5}-c_{1}-c_{2}}{2}\right)(4.5) \\
& \Gamma\left(\frac{\Delta_{3}+\Delta_{4}+c_{2}-h}{2}\right) \Gamma\left(\frac{\Delta_{1}+\Delta_{2}+c_{1}-h}{2}\right) \Gamma\left(\frac{\Delta_{3}+\Delta_{4}-c_{2}-h}{2}\right) \Gamma\left(\frac{\Delta_{1}+\Delta_{2}-c_{1}-h}{2}\right) \\
& \int \frac{\mathrm{d} \bar{s}_{1}}{\bar{s}_{1}} \frac{\mathrm{d} \bar{s}_{2}}{\bar{s}_{2}} \bar{s}_{1}^{h-c_{1}-s_{12}} \bar{s}_{2}^{h-c_{2}-s_{34}}\left(1+\bar{s}_{1}^{2}\right)^{-\delta_{35}-\delta_{45}}\left(1+\bar{s}_{2}^{2}+\bar{s}_{1}^{2} \bar{s}_{2}^{2}\right)^{-\delta_{15}-\delta_{25}}\left(1+\bar{s}_{1}^{2} \bar{s}_{2}^{2}\right)^{\frac{s_{12}-c_{1}-h}{2}} .
\end{aligned}
$$

Let us focus on the integral on the third line. After a change of variables into $x=\bar{s}_{1}^{2}, y=\bar{s}_{2}^{2}$ the integral becomes of the form

$$
\simeq \int_{0}^{+\infty} \int_{0}^{+\infty} \frac{\mathrm{d} x}{x} \frac{\mathrm{d} y}{y} x^{a} y^{b}(1+x)^{c}(1+y+x y)^{d}(1+x y)^{e}
$$

with

$$
\begin{aligned}
& a=\frac{h-c_{1}-s_{12}}{2}, \quad b=\frac{h-c_{2}-s_{34}}{2}, \quad c=-\left(\delta_{35}+\delta_{45}\right), \\
& d=-\delta_{15}-\delta_{25}, \quad e=\frac{s_{12}-c_{1}-h}{2}
\end{aligned}
$$

This integral possesses a large number of symmetries interchanging the exponents of the various factors. Rescaling $y \rightarrow y /(1+x)$ followed by $x \rightarrow 1 / x$ we obtain

$$
\int_{0}^{+\infty} \int_{0}^{+\infty} \frac{\mathrm{d} x}{x} \frac{\mathrm{d} y}{y} x^{-a+b-c} y^{b}(1+x)^{-b+c-e}(1+y)^{d}(1+x+y)^{e}
$$

To compute this integral, first perform the change of variables

$$
x \rightarrow \frac{x}{1-x}, \quad y \rightarrow \frac{y}{1-y}
$$


whereupon the integral becomes

$$
\begin{aligned}
\int_{0}^{1} & \int_{0}^{1} \frac{\mathrm{d} x}{x} \frac{\mathrm{d} y}{y} x^{-a+b-c} y^{b}(1-x)^{-1+a}(1-y)^{-1-b-d-e}(1-x y)^{e} \\
& \equiv \int_{0}^{1} \int_{0}^{1} \frac{\mathrm{d} x}{x} \frac{\mathrm{d} y}{y} x^{a_{1}} y^{a_{2}}(1-x)^{-a_{1}+b_{1}-1}(1-y)^{-a_{2}+b_{2}-1}(1-x y)^{a_{3}} .
\end{aligned}
$$

The integral can be performed assuming $\operatorname{Re}\left(b_{k}\right)>\operatorname{Re}\left(a_{k}\right)>0$, for $k=1,2$ using

$$
\begin{aligned}
& { }_{3} F_{2}\left(a_{1}, a_{2}, a_{3} ; b_{1}, b_{2} ; z\right)= \\
& \prod_{k=1}^{2} \frac{\Gamma\left(b_{k}\right)}{\Gamma\left(a_{k}\right) \Gamma\left(b_{k}-a_{k}\right)} \int_{0}^{1} \int_{0}^{1} \frac{\mathrm{d} x}{x} \frac{\mathrm{d} y}{y} x^{a_{1}} y^{a_{2}}(1-x)^{-a_{1}+b_{1}-1}(1-y)^{-a_{2}+b_{2}-1}(1-z x y)^{-a_{3}}
\end{aligned}
$$

and so we obtain

$$
\begin{aligned}
\iint(\ldots)= & \Gamma\left(\frac{c_{1}-c_{2}+\Delta_{5}}{2}\right) \Gamma\left(\frac{c_{1}+c_{2}+\Delta_{5}}{2}\right) \Gamma\left(\frac{-c_{1}+h-s_{12}}{2}\right) \Gamma\left(\frac{-c_{2}+h-s_{34}}{2}\right) \\
& \times \frac{{ }_{3} F_{2}\left(\left\{\frac{c_{1}-c_{2}+\Delta_{5}}{2}, \frac{-c_{2}+h-s_{34}}{2}, \frac{c_{1}+h-s_{12}}{2},\left\{\frac{-c_{2}+\Delta_{5}+h-s_{12}}{2}\right\}, \frac{c_{1}+\Delta_{5}+h-s_{34}}{2}\right\} ; 1\right)}{\Gamma\left(\frac{-c_{2}+\Delta_{5}+h-s_{12}}{2}\right) \Gamma\left(\frac{c_{1}+\Delta_{5}+h-s_{34}}{2}\right)} .
\end{aligned}
$$

The ${ }_{3} F_{2}$ hypergeometric function at argument $z=1$ satisfies a number of identities, among which

$$
\begin{aligned}
& { }_{3} F_{2}\left(a_{1}, a_{2}, a_{3}, b_{1}, b_{2} ; 1\right)=\frac{\Gamma\left(b_{1}\right) \Gamma\left(b_{2}\right) \Gamma\left(b_{1}+b_{2}-a_{1}-a_{2}-a_{3}\right)}{\Gamma\left(a_{1}\right) \Gamma\left(b_{1}+b_{2}-a_{1}-a_{2}\right) \Gamma\left(b_{1}+b_{2}-a_{1}-a_{3}\right)} \\
& { }_{3} F_{2}\left(b_{1}-a_{1}, b_{2}-a_{1}, b_{1}+b_{2}-a_{1}-a_{2}-a_{3}, b_{1}+b_{2}-a_{1}-a_{2}, b_{1}+b_{2}-a_{1}-a_{3} ; 1\right)
\end{aligned}
$$

which exchanges the roles of $c_{1}, s_{12}$ with $c_{2}, s_{34}$.

The expression for the Mellin amplitude is then written as

$$
\begin{aligned}
M_{5}= & \frac{g^{3}}{\Gamma\left(\frac{\Delta_{1}+\Delta_{2}-s_{12}}{2}\right) \Gamma\left(\frac{\Delta_{3}+\Delta_{4}-s_{34}}{2}\right)} \int_{-i \infty}^{+i \infty} \frac{\mathrm{d} c_{1} \mathrm{~d} c_{2}}{(2 \pi i)^{2}} \frac{L_{1}\left(c_{1}\right) L_{1}\left(-c_{1}\right)}{\left(\delta_{1}-h\right)^{2}-c_{1}^{2}} \frac{L_{2}\left(c_{2}\right) L_{2}\left(-c_{2}\right)}{\left(\delta_{2}-h\right)^{2}-c_{2}^{2}} \\
& \prod_{\sigma_{1}, \sigma_{2}= \pm 1} \Gamma\left(\frac{\sigma_{1} c_{1}+\sigma_{2} c_{2}+\Delta_{5}}{2}\right) \\
& \frac{3 F_{2}\left(\left\{\frac{c_{1}-c_{2}+\Delta_{5}}{2}, \frac{-c_{2}+h-s_{34}}{2}, \frac{c_{1}+h-s_{12}}{2}\right\},\left\{\frac{-c_{2}+\Delta_{5}+h-s_{12}}{2}, \frac{c_{1}+\Delta_{5}+h-s_{34}}{2}\right\}, 1\right)}{\Gamma\left(\frac{-c_{1}+c_{2}+\Delta_{5}}{2}\right) \Gamma\left(\frac{-c_{2}+\Delta_{5}+h-s_{12}}{2}\right) \Gamma\left(\frac{c_{1}+\Delta_{5}+h-s_{34}}{2}\right)}
\end{aligned}
$$

with

$$
L_{1}\left(c_{1}\right)=\frac{\Gamma\left(\frac{c_{1}+h-s_{12}}{2}\right) \Gamma\left(\frac{\Delta_{1}+\Delta_{2}+c_{1}-h}{2}\right)}{2 \Gamma\left(c_{1}\right)}, \quad L_{2}\left(c_{2}\right)=\frac{\Gamma\left(\frac{c_{2}+h-s_{34}}{2}\right) \Gamma\left(\frac{\Delta_{3}+\Delta_{4}+c_{2}-h}{2}\right)}{2 \Gamma\left(c_{2}\right)} .
$$

The identity between these two expressoins will be shown in the next section. We are interested in obtaining the poles and respective residues in $s_{12}$ and $s_{34}$ of the expression above. Although there are various sets of poles in $c_{1}$ and $c_{2}$, the only ones which will end 


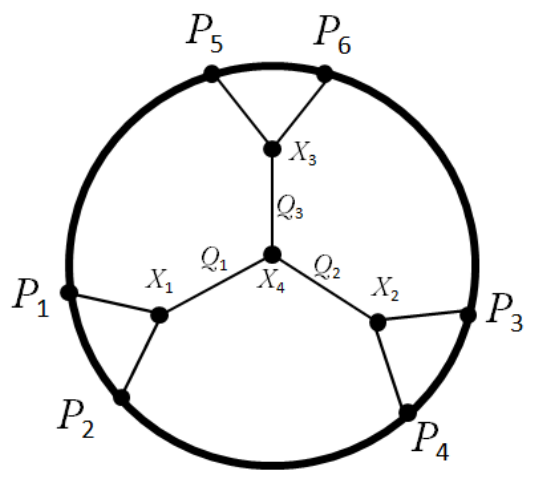

Figure 6. A six-point Witten diagram in scalar theory.

up giving expressions containing poles in $s_{12}$ and $s_{34}$ are the ones at $c_{1}=\delta_{1}-h, c_{2}=\delta_{2}-h$. Computing the residues at these poles we find

$$
M_{5}=\sum_{n_{1}, n_{2}=0}^{+\infty} \frac{P_{n_{1}}^{\delta_{1}}}{s_{12}-\delta_{1}-2 n_{1}} \frac{P_{n_{2}}^{\delta_{2}}}{s_{34}-\delta_{2}-2 n_{2}} V_{\left[0,0, n_{1}\right]}^{\Delta_{1}, \Delta_{2}, \delta_{1}} V_{\left[0,0, n_{2}\right]}^{\Delta_{3}, \Delta_{4}, \delta_{2}} V_{\left[0, n_{1}, n_{2}\right]}^{\Delta_{5}, \delta_{1}, \delta_{2}}+\ldots
$$

where the dots represent possible subleading contributions. The only new ingredient in the above is

$$
\begin{aligned}
V_{\left[0, n_{1}, n_{2}\right]}^{\Delta_{1}, \Delta_{2}, \Delta_{3}}= & g \Gamma\left(\frac{\sum_{i} \Delta_{i}-2 h}{2}\right)\left(1-\frac{1}{2} \sum_{i}^{3} \Delta_{i}+\Delta_{2}\right)_{n_{1}}\left(1-\frac{1}{2} \sum_{i}^{3} \Delta_{i}+\Delta_{3}\right)_{n_{2}} \times \quad(4.17) \\
& { }_{3} F_{2}\left(\left\{\frac{\sum_{i} \Delta_{i}-2 h}{2},-n_{1},-n_{2}\right\},\left\{\frac{\sum_{i} \Delta_{i}-2 \Delta_{2}-2 n_{1}}{2}, \frac{\sum_{i} \Delta_{i}-2 \Delta_{3}-2 n_{2}}{2}\right\}, 1\right)
\end{aligned}
$$

It is easy to check that when one or more of the $n_{i}$ 's vanish we reproduce our previous expressions (3.24), (3.25). It's been a long way, but the final result (4.16) is particularly simple, and it agrees with the Feynman rules we have defined previously, assuming that the subleading contributions in the above vanish. Attempts to evaluate the Mellin amplitude numerically suggest this is the case, although further work is necessary. The upshot of this calculation is that we have now in our possession a further ingredient for such rules, which is the vertex for the case where we have two "descendant" fields and one primary.

\subsection{6-point amplitude}

The next step is to calculate a six point diagram involving three bulk-to-bulk propagators connected at a single vertex in order to obtain $V_{\left[n_{1}, n_{2}, n_{3}\right]}^{\Delta_{1}, \Delta_{2}, \Delta_{3}}$. With this purpose in mind we now turn our attention to the particular Witten diagram in figure 6. We can immediately write

$$
A_{6}=\int_{-i \infty}^{+i \infty} \prod_{k=1}^{3} \frac{\mathrm{d} c_{k}}{2 \pi i} f_{\delta_{k}}\left(c_{k}\right) \int_{\partial \mathrm{AdS}} \prod_{i=1}^{3} \mathrm{~d} Q_{i} A\left(1,2, c_{1}^{+}\right) A\left(3,4, c_{2}^{+}\right) A\left(5,6, c_{3}^{+}\right) A\left(c_{1}^{-}, c_{2}^{-}, c_{3}^{-}\right)
$$

The calculation proceeds as for the five point amplitude - we integrate over each $Q_{i}$ in turn. Exactly as before one can use the Symanzik star formula to read off the Mellin 
amplitude. After performing the $s_{1}, s_{2}, s_{3}$ integrals (just like before we could immediately do the integrals in $s_{1}$ and $s_{2}$ ), we are still left with a seemingly complicated integral in $\bar{s}_{1}, \bar{s}_{2}, \bar{s}_{3}$, analogous to the second line of (4.5). However, as we show in appendix C, performing a change of variables it is possible to write the Mellin amplitude as

$$
\begin{aligned}
M_{6}= & \frac{g^{4}}{2^{6}} \int_{-i \infty}^{i \infty} \prod_{i=1}^{3}\left(\frac{\mathrm{d} c_{i}}{2 \pi i} \frac{\Gamma\left(\frac{\Delta_{i, 1}+\Delta_{i, 2}+c_{i}-h}{2}\right) \Gamma\left(\frac{\Delta_{i, 1}+\Delta_{i, 2}-c_{i}-h}{2}\right) \Gamma\left(\frac{c_{i}+h-s_{i}}{2}\right)}{\Gamma\left(\frac{\Delta_{i, 1}+\Delta_{i, 2}-s_{i}}{2}\right) \Gamma\left(c_{i}\right) \Gamma\left(-c_{i}\right)\left[\left(\delta_{i}-h\right)^{2}-c_{i}^{2}\right]}\right) \\
& \Gamma\left(\frac{h-c_{1}-c_{2}-c_{3}}{2}\right) \int_{0}^{+\infty} \frac{\mathrm{d} x}{x} \frac{\mathrm{d} y}{y} \frac{\mathrm{d} z}{z} x^{a} y^{b} z^{c}(1+x)^{d}(1+y)^{e}(1+z)^{f}(1+x+y+z)^{g},
\end{aligned}
$$

with $\Delta_{i, j}$ the dimension of the $j$ th field of the $i$ th pair of legs - $j=1,2$ and $i=1,2,3$. For instance, $\Delta_{2,1} \equiv \Delta_{3}, \Delta_{3,2} \equiv \Delta_{6}, \ldots$. Also, the $s_{i}$ variables are the Mandelstam variables associated with each pair of legs, such that $s_{1} \equiv s_{12}, s_{2} \equiv s_{34}$ and $s_{3} \equiv s_{56}$. As for the parameters $a, b, \ldots, g$ we have $g=\frac{1}{2}\left(c_{1}+c_{2}+c_{3}-h\right)$ and

$$
\begin{aligned}
& a=\frac{1}{2}\left(-c_{1}+h-s_{12}\right), \quad b=\frac{1}{2}\left(-c_{2}+h-s_{34}\right) \quad c=\frac{1}{2}\left(-c_{3}+h-s_{56}\right) \\
& d=\frac{1}{2}\left(-c_{1}-h+s_{12}\right), \quad e=\frac{1}{2}\left(-c_{2}-h+s_{34}\right), \quad f=\frac{1}{2}\left(-c_{3}-h+s_{56}\right),
\end{aligned}
$$

To proceed we must evaluate the integral on the second line of (4.19). First we do a multinomial expansion on the last factor of the integrand,

$$
\begin{aligned}
(1+x+y+z)^{g}= & \sum_{m_{1}, m_{2}, m_{3}=0}^{+\infty}(-g)_{m_{1}}\left(-g+m_{1}\right)_{m_{2}}\left(-g+m_{1}+m_{2}\right)_{m_{3}} \\
& \times \frac{(-x)^{m_{1}}}{m_{1} !} \frac{(-y)^{m_{2}}}{m_{2} !} \frac{(-z)^{m_{3}}}{m_{3} !}
\end{aligned}
$$

We are then free to perform the separate integrations over $x, y, z$. The result is

$$
\int_{0}^{+\infty} \int_{0}^{+\infty} \int_{0}^{+\infty}(\ldots)=\prod_{i=1}^{3} \frac{\Gamma\left(c_{i}\right) \Gamma\left(\frac{-c_{i}+h-s_{i}}{2}\right)}{\Gamma\left(\frac{c_{i}+h-s_{i}}{2}\right)} F_{A}^{(3)}(-g,\{a, b, c\},\{d, e, f\} ; 1,1,1)
$$

where $s_{1}=s_{12}, \ldots$ and $F_{A}^{(3)}$ is a Lauricella generalized hypergeometric function of three variables [26-28]. For future reference we give the definition of the Lauricella function $F_{A}^{(m)}$ :

$$
F_{A}^{(m)}\left(g,\left\{a_{1}, \ldots, a_{m}\right\},\left\{b_{1}, \ldots, b_{m}\right\} ; x_{1}, \ldots, x_{m}\right) \equiv \sum_{n_{i}=0}^{+\infty}\left((g)_{\sum_{i=1}^{m} n_{i}} \prod_{i=1}^{m} \frac{\left(a_{i}\right)_{n_{i}}}{\left(b_{i}\right)_{n_{i}}} \frac{x_{i}^{n_{i}}}{n_{i} !}\right)
$$

The above series is convergent only for $\sum_{i}\left|x_{i}\right|<1$. Our interpretation then is to define the sum at this point as the value of the Lauricella function at that point, which is well defined via analytic continuation. Of course it might very well happen that for specific values of the parameters $g, a_{i}, b_{i}$ the series reduces to a sum, in which case everything is perfectly well defined. 
The Mellin amplitude is exactly given by

$$
\begin{aligned}
M_{6}= & g^{4} \int_{-i \infty}^{i \infty} \prod_{i=1}^{3}\left(\frac{\mathrm{d} c_{k}}{2 \pi i} \frac{L_{i}\left(c_{i}\right) L_{i}\left(-c_{i}\right)}{\left(\delta_{i}-h\right)^{2}-c_{i}^{2}}\right) \Gamma\left(\frac{h-c_{1}-c_{2}-c_{3}}{2}\right) \\
& \prod_{i=1}^{3}\left(\frac{\Gamma\left(c_{i}\right)}{\Gamma\left(\frac{\Delta_{i, 1}+\Delta_{i, 2}-s_{i}}{2}\right) \Gamma\left(\frac{c_{i}+h-s_{i}}{2}\right)}\right) F_{A}^{(3)}(-g,\{a, b, c\},\{d, e, f\} ; 1,1,1) .
\end{aligned}
$$

with the $L_{i}$ defined analogously to (4.15). Evaluating the integral above in closed form seems like a difficult challenge. The poles in $s_{12}, s_{34}, s_{56}$ however, are easily found by pinching of two poles in the $c_{1}, c_{2}$ and $c_{3}$ integrations respectively, using the definition (4.23) of $F_{A}^{(3)}$. The end result is the remarkably simple expression

$$
M_{6}=\sum_{n_{1}, n_{2}, n_{3}=0}^{+\infty}\left(\prod_{i=1}^{3} \frac{P_{n_{i}}^{\delta_{i}}}{s_{i}-\delta_{i}-2 n_{i}}\right) V_{\left[0,0, n_{1}\right]}^{\Delta_{1}, \Delta_{2}, \delta_{1}} V_{\left[0,0, n_{2}\right]}^{\Delta_{3}, \Delta_{4}, \delta_{2}} V_{\left[0,0, n_{3}\right]}^{\Delta_{5}, \Delta_{6}, \delta_{3}} V_{\left[n_{1}, n_{2}, n_{3}\right]}^{\delta_{1}, \delta_{2}, \delta_{3}}+\ldots
$$

This not only provides further evidence for our set of Feynman rules for Mellin amplitudes, but also gives us the final vertex

$$
\begin{gathered}
V_{\left[n_{1}, n_{2}, n_{3}\right]}^{\Delta_{1}, \Delta_{2}, \Delta_{3}}=V_{[0,0,0]}^{\Delta_{1}, \Delta_{2}, \Delta_{3}}\left(1-h+\Delta_{1}\right)_{n_{1}}\left(1-h+\Delta_{2}\right)_{n_{2}}\left(1-h+\Delta_{3}\right)_{n_{3}} \\
F_{A}^{(3)}\left(\frac{\Delta_{1}+\Delta_{2}+\Delta_{3}-2 h}{2},\left\{-n_{1},-n_{2},-n_{3}\right\},\left\{1+\Delta_{1}-h, 1+\Delta_{2}-h, 1+\Delta_{3}-h\right\} ; 1,1,1\right) .
\end{gathered}
$$

Notice that with $n_{i}$ positive integers, the Lauricella triple hypergeometric function is given by a finite sum.

Now let us show that the vertex function $V_{\left[n_{1}, n_{2}, n_{3}\right]}^{\Delta_{1}, \Delta_{2}, \Delta_{3}}$ just computed reduces to the previous expression (4.17) when one of the integers $n_{i}$ is zero. When this happens, one of the sums in the definition (4.23) reduces to a single term, and the Lauricella triple hypergeometric function reduces to the Appell $F_{2}$ function, which we denote by $F_{A}^{(2)}$. For instance, if $n_{3}=0$ we get

$$
\begin{aligned}
V_{\left[n_{1}, n_{2}, n_{3}\right]}^{\Delta_{1}, \Delta_{2}, \Delta_{3}}= & V_{[0,0,0]}^{\Delta_{1}, \Delta_{2}, \Delta_{3}}\left(1-h+\Delta_{1}\right)_{n_{1}}\left(1-h+\Delta_{2}\right)_{n_{2}} \\
& F_{A}^{(2)}\left(\frac{\Delta_{1}+\Delta_{2}+\Delta_{3}-2 h}{2},\left\{-n_{1},-n_{2}\right\},\left\{1+\Delta_{1}-h, 1+\Delta_{2}-h\right\} ; 1,1\right) .
\end{aligned}
$$

The Appell $F_{2}$ function with arguments $x=y=1$ is directly related to the ${ }_{3} F_{2}$ hypergeometric function at argument $x=1$. In order to prove this one computes the integral

$$
\int_{0}^{+\infty} \int_{0}^{+\infty} \frac{\mathrm{d} x}{x} \frac{\mathrm{d} y}{y} x^{-a+b-c} y^{b}(1+x)^{-b+c-e}(1+y)^{d}(1+x+y)^{e}
$$

in two different ways, firstly by using formula (4.11), and secondly using the multinomial expansion on $(1+x+y)^{e}$ and integrating. The end result is

$$
\begin{aligned}
& F_{A}^{(2)}(e,\{a, b\},\{c, d\} ; 1,1)= \\
& \quad \frac{(1+a-c+e)_{-a}(1+b-d+e)_{-b}}{(1+a-c)_{-a}(1+b-d)_{-b}}{ }_{3} F_{2}(\{a, b, e\},\{1+a-c+e, 1+b-d+e\}, 1)
\end{aligned}
$$

Using this identity it is straightforward to show that (4.27) reduces to (4.17). 


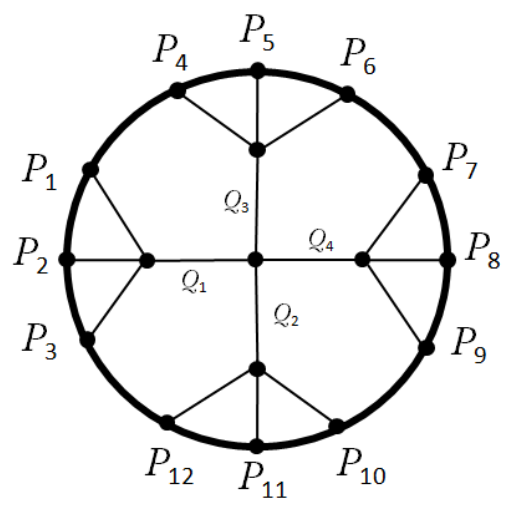

Figure 7. A twelve-point diagram in $\phi^{4}$ theory.

\subsection{Outline of the 12-point amplitude calculation}

The beautiful expression (4.26) for the general vertex $V_{\left[n_{1}, n_{2}, n_{3}\right]}^{\Delta_{1}, \Delta_{2}, \Delta_{3}}$ in $\phi^{3}$ theory leads us to conjecture that in $\phi^{m}$ theory the general vertex takes the form given in the introduction,

$$
\begin{aligned}
V_{\left[n_{1}, \ldots, n_{m}\right]}^{\Delta_{1} \ldots \Delta_{m}}= & g_{m} \Gamma\left(\frac{\sum_{i} \Delta_{i}-2 h}{2}\right)\left(\prod_{i=1}^{m}\left(1-h+\Delta_{i}\right)_{n_{i}}\right) \\
& F_{A}^{(m)}\left(\frac{\sum_{i=1}^{n} \Delta_{i}-2 h}{2},\left\{-n_{1}, \ldots,-n_{m}\right\},\left\{1+\Delta_{1}-h, \ldots, 1+\Delta_{m}-h\right\} ; 1, \ldots, 1\right) .
\end{aligned}
$$

As a rather non-trivial check of this, we have performed the computation of a twelvepoint amplitude in $\phi^{4}$ theory. The calculation is tedious but essentially the same as in the six point function in $\phi^{3}$ theory. The diagram is of the form given in figure 7 . The computation of this amplitude is very similar to the six-point calculation. The $X$ integrals are performed trivially as usual. The integrals over boundary coordinates $Q_{i}$ are also trivial, and the resulting expression can be translated into a Mellin amplitude consisting of four $c_{i}$ integrals, and a set of four $s_{i}, \bar{s}_{i}$ integrals. The latter can be explicitly performed, while the former lead to poles in the various Mandelstam variables upon pinching. The $s_{i}$ integrals can be carried out immediately as in the four-, five- and six-point amplitude calculations, so that the only non-trivial part of the calculations are the remaining integrals over the $\bar{s}_{i}$ parameters. At this point we are in a situation similar to that described in appendix (C), with a rather nasty looking integrand. However, by performing change of variables of the type described in that same appendix, the integral can be successively simplified until it reduces to

$$
\int_{0}^{+\infty} \prod_{i=1}^{4}\left[\mathrm{~d} x_{i} x_{i}^{a_{i}}(1+x)^{b_{i}}\right]\left(1+\sum_{i}^{4} x_{i}\right)^{g}
$$

with $g=\frac{1}{2}\left(c_{1}+c_{2}+c_{3}+c_{4}-h\right)$,

$$
a_{i}=\frac{1}{2}\left(-c_{i}+h-m_{i}\right), \quad b_{i}=\frac{1}{2}\left(-c_{i}-h+m_{i}\right)
$$


and the four Mandelstam variables $m_{i}$ are $m_{1} \equiv s_{123}, m_{2} \equiv s_{456}, \ldots$. To evaluate the integral we perform a multinomial expansion as before, which leads to the four-variable Lauricella function. The calculation then proceeds as for the six-point function and one precisely finds an expression for the poles of the Mellin amplitude consistent with the Feynman rules conjectured in the introduction.

\section{Conformal invariance of index structure}

In the following sections we will be interested in evaluating amplitudes which involve fields carrying spin degress of freedom, either in an internal propagator or as an external state. In the latter case, to obtain expressions for amplitudes in $d$-dimensional space, we will have to contract the $M$ indices with the pull-backs $\zeta_{\mu}^{M}$. These in turn are contracted with some polarization tensors, so that overall we may say that the $M$ indices are contracted with polarizations $\xi^{M}$. These polarizations satisfy

$$
\xi_{1} \cdot P_{1}=\xi_{1}^{M_{1}} P_{1, M_{1}}=\xi^{\mu} \frac{\partial P_{1}^{M_{1}}}{\partial y_{1}^{\mu}} P_{1, M_{1}}=0
$$

because of the condition $P_{1}^{2}=0$. Further, we have

$$
P_{M} D_{\Delta}^{M A}=P^{A}\left(1+\frac{1}{\Delta} P^{M} \partial_{M}\right)=0
$$

The rightmost factor checks that the overall amplitude scales with $P$ like $1 / P^{\Delta}$, which has to be the case, and so it is vanishing. That is, the transversality condition of $J^{M A}$ has transformed into a scaling condition imposed by $D^{M A}$. In this way, $D^{M A}$ can be thought of as a projector which implements conformal symmetry of the index structure.

Overall, these results are very suggestive: in the embedding formalism, amplitudes depend on objects $P_{i}$ and polarizations $\xi_{i}$ such that

$$
P_{i}^{2}=0, \quad \xi_{i} \cdot P_{i}=0, \quad \xi_{i} \simeq \xi_{i}+P_{i}
$$

These are exactly the conditions required of a gauge theory amplitude depending on momenta $P_{i}$ and polarizations $\xi_{i}$. This suggests that $d$-dimensional CFT dynamics are related to gauge (or gravity) theories in $d+2$ dimensions, but where the coordinates of the one are related to the momenta of the other. Although we will not try to flesh out this relation further here, the above set of requirements above already imply strong constraints on the possible index structure of conformally invariant amplitudes.

Consider for instance an amplitude of the form $\left\langle J_{3}^{M}\left(P_{3}\right) \mathcal{O}\left(P_{1}\right) \mathcal{O}\left(P_{2}\right)\right\rangle$. On the one hand, no $P_{3}$ with free indices are allowed, so that the index dependence must be carried by $P_{1}, P_{2}$. Then "gauge invariance" uniquely fixes the structure

$$
\frac{P_{1}^{M_{3}}}{P_{13}}-\frac{P_{2}^{M_{3}}}{P_{23}} \equiv X_{12}^{M_{3}} .
$$

The rest of the amplitude is fixed by requiring the correct behaviour under rescalings of $P_{1}, P_{2}, P_{3}$ by constant factors. Generically, the only structures which can appear in any 
amplitude are of the form above or

$$
I^{M_{1} M_{2}} \equiv \eta^{M_{1} M_{2}}-\frac{P_{1}^{M_{2}} P_{2}^{M_{1}}}{P_{1} \cdot P_{2}}
$$

which vanishes upon contraction with either $P_{1}^{M_{1}}$ or $P_{2}^{M_{2}}$.

In particular, consider a current three-point function. The general structure of such amplitudes, as imposed by conformal invariance has been known for a long time. With our methods, finding the index structure of such an amplitude is a trivial task: there are only two possible structures, namely

$$
X_{23}^{M_{1}} X_{13}^{M_{2}} X_{12}^{M_{3}}, \quad \text { or } \quad I^{M_{1} M_{2}} X_{12}^{M_{3}}+\text { permutations }
$$

And indeed, this is correct. A similar argument can be made for the four-point function. All terms are of the form

$$
I I, \quad I X X, \quad X X X X
$$

but there are a greater number of them, as one could have several $X^{\prime} s$ with the same index, e.g. $X_{12}^{M_{4}}, X_{13}^{M_{4}}, X_{23}^{M_{4}}$.

The arguments given above are completely general, in the sense that they apply to any conformal correlation function independently of the spin or number of fields involved. In other words, the most general amplitude must have an index structure such that it reduces to polynomials in $I, X$. In general, current conservation places constraints on the final form of the amplitude by relating the coefficients of different kinds of index structures. However such constraints do not seem to have a simple formulation in the embedding formalism, and they are most usefully seen by pulling back our expressions to $d$-dimensions.

Actually there is a slight subtlety we have ommitted. It is easiest to see the problem in the case of the stress-tensor. This is the question of removal of traces from the index structure, which can be understood by the simple example of the correlator of a stresstensor and two scalar fields. The index structure of such a correlator is completely fixed by conformal invariance, ${ }^{6}$ and we get

$$
\left\langle T^{M_{3} N_{3}}\left(P_{3}\right) \mathcal{O}\left(P_{1}\right) \mathcal{O}\left(P_{2}\right)\right\rangle \propto X_{12}^{M_{3}} X_{12}^{N_{3}}-\text { trace. }
$$

The question is, what exactly do we mean by the trace part removal in the above? If we remove the $(d+2)$ dimensional trace of the expression above, so that it becomes

$$
\simeq X_{12}^{M_{3}} X_{12}^{N_{3}}-\frac{1}{d+2} \eta^{M_{3} N_{3}}\left(X_{12}\right)^{2}
$$

then we lose "gauge invariance" as easily seen. Another problem is that it is $1 /(d+2)$ which appears in the expression, whereas we expect the final result to be traceless in $d$ dimensions, not $d+2$. As it turns out, both these problems can be solved at once. To restore gauge invariance we must, counter-intuitively, add gauge-variant terms. To do this, first introduce introduce the vector $Q$ which in our parameterization is simply

\footnotetext{
${ }^{6}$ See for instance [18].
} 
$Q=\left(Q^{+}, Q^{-}, Q_{\mu}\right)=(0,1,0)$. This implies that $Q \cdot P=-1 / 2$, for any boundary point $P$. Then to remove the trace we take

$$
\simeq X_{12}^{M_{3}} X_{12}^{N_{3}}-\frac{1}{d}\left(\eta^{M_{3} N_{3}}+4 P^{\left(M_{3}\right.} Q^{\left.N_{3}\right)}\right)\left(X_{12}\right)^{2},
$$

It is easily checked that the expression above is both gauge-invariant and traceless, at least in $d+2$ dimensions. Also, the extra terms we have introduced vanish upon contraction with the pull-backs $\zeta_{\mu}^{M}$, and we obtain an expression which is traceless in $d$ dimensions.

This result is more general, and it applies to any pair of symmetric traceless indices. It follows from [24]

$$
\eta^{\mu \nu} \zeta_{\mu}^{M_{1}}(P) \zeta_{\nu}^{M_{2}}(P) T_{M_{1} M_{2} \ldots}(P)=\eta^{M_{1} M_{2}} T_{M_{1} M_{2} \ldots}(P), \quad \text { if } \quad P_{1}^{M} T_{M_{1} \ldots}(P)=0
$$

which is easily proved noting that in our parameterization we have

$$
\eta^{\mu \nu} \zeta_{\mu}^{M_{1}} \zeta_{\nu}^{M_{2}}=\left(\eta_{M_{1} M_{2}}+4 Q^{\left(M_{1}\right.} P^{\left.M_{2}\right)}\right) .
$$

With these results, we may say that before taking traces, the amplitude is indeed fully written in terms of the objects $I^{M N}, X_{i j}^{N}$ defined previously.

\section{Current amplitudes}

\section{$6.1\langle J \mathcal{O O}\rangle$ correlator}

To begin this section, we shall compute the three-point function of a current with two scalar operators using the embedding formalism. While the final result is well known, this calculation will serve to illustrate the usage of the embedding formalism for the computation of current amplitudes. Also, as we shall see in the next section, it will immediately give us the result for the three current amplitude.

We take for the gravitational action that of a minimally coupled scalar of mass $m^{2}=$ $\Delta(\Delta-d)$

$$
S=\int \mathrm{d}^{d+1} x \sqrt{-g}\left(-\frac{1}{4} F_{M N} F^{M N}+\left|\nabla_{M} \phi-i e A_{M} \phi\right|^{2}+m^{2} \phi^{2}\right) .
$$

The three point vertex is of the form

$$
\text { ie } A_{M}\left(P_{3}\right)\left(\nabla \phi\left(P_{1}\right) \phi\left(P_{2}\right)-\nabla \phi\left(P_{2}\right) \phi\left(P_{1}\right)\right) \text {. }
$$

The amplitude is therefore

$$
\left\langle J^{M} \mathcal{O} \mathcal{O}\right\rangle=2 i e D_{d-1}^{M_{3} A} \int \prod_{i=1}^{3} \frac{\mathrm{d} t_{i}}{t_{i}} t_{i}^{\Delta_{i}} \int_{\mathrm{AdS}} \mathrm{d} X\left(t_{1} P_{1, A}-t_{2} P_{2, A}\right) \exp \left[2\left(t_{1} P_{1}+t_{2} P_{2}+t_{3} P_{3}\right) \cdot X\right]
$$

with $\Delta_{1}=\Delta_{2} \equiv \Delta$. Recall that $D_{d-1}^{M A}$ is an operator which acts on the right-hand side of the expression. After the $X$ integration we obtain

$$
\left\langle J^{M} \mathcal{O O}\right\rangle=2 i e \pi^{h} \Gamma\left(\frac{\sum_{i} \Delta_{i}+1-2 h}{2}\right) D_{d-1}^{M_{3} A} \int \prod_{i=1}^{3} \frac{\mathrm{d} t_{i}}{t_{i}} t_{i}^{\Delta_{i}}\left(t_{1} P_{1, A}-t_{2} P_{2, A}\right) e^{-\sum_{i<j}^{3} t_{i} t_{j} P_{i j}} .
$$


Let us focus on the integral. This is quite similar to the one we found for the scalar three point function, and we can proceed using a trick:

$$
\begin{gathered}
\int \prod_{i=1}^{3} \frac{\mathrm{d} t_{i}}{t_{i}} t_{i}^{\Delta_{i}}\left(t_{1} P_{1, A}\right) e^{-\sum_{i<j}^{3} t_{i} t_{j} P_{i j}}=\int \prod_{i=1}^{2} \frac{\mathrm{d} t_{i}}{t_{i}} t_{i}^{\Delta_{i}} \frac{\mathrm{d} t_{3}}{t_{3}} t_{3}^{d-2}\left(t_{3} t_{1} P_{1, A}\right) e^{-\sum_{i<j}^{3} t_{i} t_{j} P_{i j}} \\
\quad=-P_{1, A} \frac{\partial}{\partial P_{13}} \int \prod_{i=1}^{2} \frac{\mathrm{d} t_{i}}{t_{i}} t_{i}^{\Delta_{i}} \frac{\mathrm{d} t_{3}}{t_{3}} t_{3}^{d-2} e^{-\sum_{i<j}^{3} t_{i} t_{j} P_{i j}}=\frac{\delta_{13}}{2} \frac{P_{1, A}}{P_{13}} \prod_{i<j} \Gamma\left(\delta_{i j}\right)\left(P_{i j}\right)^{-\delta_{i j}} .
\end{gathered}
$$

In the last expression, the $\delta_{i j}$ satisfy the constraint $\sum_{i \neq j} \delta_{i j}=\Delta_{j}-s_{j}$, with $s_{j}$ the spin of the field $j$. Overall, we get

$$
\left\langle J^{M} \mathcal{O O}\right\rangle=i e \pi^{h} \Gamma\left(\frac{\sum_{i} \Delta_{i}+1-2 h}{2}\right) D_{d-1}^{M_{3} A}\left(\delta_{13} \frac{P_{1, A}}{P_{13}}-\delta_{23} \frac{P_{2, A}}{P_{23}}\right) \prod_{i<j} \Gamma\left(\delta_{i j}\right)\left(P_{i j}\right)^{-\delta_{i j}}
$$

To finish, we are left with the action of the operator $D_{d-1}^{M A}$. However, this action is particularly simple here. To see this, first write

$$
D_{d-1}^{M A}=\eta^{M_{3} A}+\frac{1}{d-1} P_{3}^{A} \partial_{M_{3}}=\frac{d-2}{d-1} \eta^{M_{3} A}+\frac{1}{d-1} \frac{\partial}{\partial P_{3}^{M_{3}}} P_{3}^{A}
$$

Since we have $\delta_{13}=\delta_{23}=\frac{1}{2}(d-2)$, the second term in the operator leads to a vanishing result. Restoring the external leg normalizations the final answer is

$$
\left\langle J^{M_{3}} \mathcal{O O}\right\rangle=\operatorname{ie} C\left(\frac{P_{1}^{M_{3}}}{P_{13}}-\frac{P_{2}^{M_{3}}}{P_{23}}\right) \prod_{i<j}\left(P_{i j}\right)^{-\delta_{i j}}
$$

with

$$
C=\frac{1}{4 \pi^{2 h}} \frac{\Gamma(h) \Gamma(\Delta)}{\Gamma(1+\Delta-h)^{2}}
$$

Following our general discussion in section (5) we have $P_{3}^{M_{3}}\left\langle J^{M_{3}} \mathcal{O O}\right\rangle=0$, and the index structure is indeed of the form $X_{12}^{M_{3}}$ as expected.

\subsection{Current three-point amplitude}

We now consider a three point amplitude of a non-abelian Yang-Mills field in AdS, or alternatively, the conformal correlation function of three currents valued in some Lie algebra with structure constants $f_{a b c}$. The Witten diagram is essentially same as in figure 2. As usual, the $X$ integration is trivial and we can immediately write

$$
\begin{aligned}
\left\langle J^{a, M_{1}}\left(P_{1}\right) J^{b, M_{2}}\left(P_{2}\right) J^{c, M_{3}}\left(P_{3}\right)\right\rangle & =i \text { e }\left(2 \pi^{h}\right) \Gamma(d-1) f^{a b c} D^{M_{1} A} D^{M_{2} B} D^{M_{3} C} I_{A B C}, \\
I_{A B C} & =\int \prod_{i=1}^{3} \frac{\mathrm{d} t_{i}}{t_{i}} t_{i}^{\Delta_{i}}\left[\eta_{A B}\left(t_{1} P_{1, C}-t_{2} P_{2, C}\right)+\text { perms }\right] e^{-\sum_{i<j} t_{i} t_{j} P_{i j}} .
\end{aligned}
$$

This expression is remarkable, in that most of the complicated index structure has effectively been moved to the action of the $D$ operators. Each of the permutations inside 
the integral sign is essentially nothing but the three point function of a current with two scalars, which we have already computed! Therefore we can immediately write down

$$
I^{A B C}=\frac{(d-2)^{2}}{2}\left(\frac{X_{12}^{C} \eta^{A B}}{P_{12}}+\text { perms }\right) \prod_{i<j} \Gamma\left(\delta_{i j}\right)\left(P_{i j}\right)^{-\delta_{i j}} .
$$

where $\delta_{i j}=\frac{1}{2}(d-2)$. To obtain the full amplitude one has but to mechanically act with the $D$ operators on the expression. Before we do this however, it is worth noticing the simplicity of the expression between parenthesis, which bears an uncanny resemblance to a gauge theory amplitude:

$$
\left(\frac{P_{1}^{C}}{P_{13}}-\frac{P_{2}^{C}}{P_{23}}\right) \frac{\eta^{A B}}{P_{12}} \rightarrow\left(k_{1}^{c}-k_{2}^{c}\right) \eta^{a b}
$$

Also, we haven't defined what the Mellin amplitude should be for the case of amplitudes involving currents. A natural definition seems to be that one should take as the Mellin amplitude the expression between parenthesis, since once this is given the entire real space amplitude may be determined after the action of the $D$ operators.

As a check that we haven't made a mistake, we may evaluate the full amplitude by acting with the $D$ operators. After some work one obtains:

$$
\begin{aligned}
&\left\langle J^{a, M_{1}}\left(P_{1}\right) J^{b, M_{2}}\left(P_{2}\right) J^{c, M_{3}}\left(P_{3}\right)\right\rangle= \\
& C_{3}\left[\left(\frac{I^{M_{1} M_{2}} X_{12}^{M_{3}}}{P_{12}}+\text { perms }\right)-\frac{3}{2} \frac{d-2}{2 d-3} X_{12}^{M_{3}} X_{23}^{M_{1}} X_{31}^{M_{2}}\right] \prod_{i<j}^{3}\left(P_{i j}\right)^{-\delta_{i j}}
\end{aligned}
$$

with

$$
C_{3}=\frac{i e}{8 \pi^{h}} f^{a b c} \Gamma(d-1) \frac{(2 d-3)(d-2)}{(d-1)^{3}} .
$$

This agrees with previous results in the literature [7] up to normalization conventions. Also as expected, the full amplitude is a polynomial in $I, X$, and satisfies the "gauge invariance" condition. This calculation shows how the embedding formalism simplifies considerably the calculation of the amplitudes.

\subsection{Scalar 4-point with current exchange}

In this section we will be computing the contribution to the scalar 4-point function of a diagram where a gauge boson is being exchanged. This will be useful as practice to the calculation of the 4-current amplitude in the next section. It will also allow us to check our formalism is correct by checking that the pole structure of the Mellin amplitude agrees with the general results of Mack [19].

The process we'll be considering is described by the Witten diagram in figure 8 . The gauge-boson bulk-to-bulk propagator can be written as a product of two bulk-to-boundary propagators, and we can write

$$
A_{4}^{J}=\int \frac{\mathrm{d} c}{2 \pi i} f_{\delta}^{1}(c) \int \mathrm{d} Q\left\langle J_{h+c}^{M}(Q) \mathcal{O}\left(P_{1}\right) \mathcal{O}\left(P_{2}\right)\right\rangle \eta_{M N}\left\langle J_{h-c}^{N}(Q) \mathcal{O}\left(P_{3}\right) \mathcal{O}\left(P_{4}\right)\right\rangle
$$




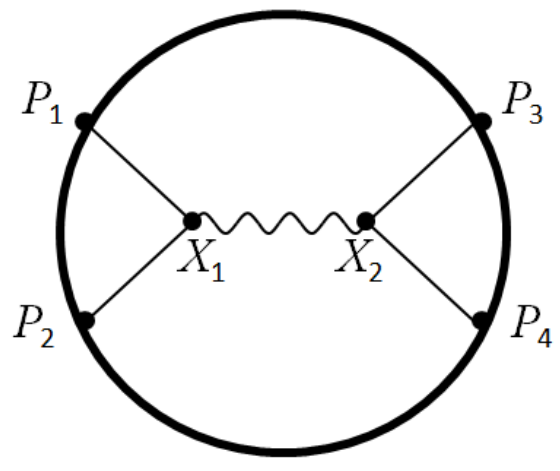

Figure 8. Gauge boson exchange diagram.

We have already computed the three-point functions appearing in the expression above. However, in practice one does not want work with the three-point function, but rather with its Schwinger parameterized form, as to be able to perform the $Q$ integral.

Notice that in the three point functions above, the currents $J$ have conformal dimensions $h \pm c$, and not $d-1$ as usual; that is

$$
\begin{aligned}
& \left\langle J_{h \pm c}^{M}(Q) \mathcal{O}\left(P_{1}\right) \mathcal{O}\left(P_{2}\right)\right\rangle= \\
& 2 i e \pi^{h} D_{h \pm c}^{M A} \int \frac{\mathrm{d} t_{1}}{t_{1}} \frac{\mathrm{d} t_{2}}{t_{2}} \frac{\mathrm{d} s}{s} t_{1}^{\Delta_{1}} t_{2}^{\Delta_{2}} s^{h+c}\left(t_{1} P_{1, A}-t_{2} P_{2, A}\right) \exp \left[-t_{1} t_{2} P_{12}+2 s\left(t_{1} P_{1}+t_{2} P_{2}\right) \cdot Q\right]
\end{aligned}
$$

In order to perform the $Q$ integrals, we need to do something about the $Q$ and $Q$ derivative hidden in the $D$ operators. However, as in the calculation of $\langle J \mathcal{O O}\rangle$ amplitude, we can write

$$
D_{h \pm c}^{M A}=\frac{h \pm c-1}{h \pm c} \eta^{M A}+\frac{\partial}{\partial Q^{M}} Q^{A},
$$

and, as before, the second term does not contribute. Each $D$ operator reduces to a Minkowski metric times a factor, and the contraction of both of them leads to

$$
\eta_{M N} D^{M A} D^{N B} \rightarrow \frac{(h-1)^{2}-c^{2}}{h^{2}-c^{2}} \eta^{A B}
$$

The prefactor in the above exactly cancels a similar factor in the definition of $f_{\delta, 1}(c)$, reducing it to $f_{\delta, 0}(c)$ (c.f. equation (2.20)). The $Q$ integrations proceed as in the scalar exchange computation of section 3.2 , and we get

$$
\begin{aligned}
A_{4}^{J}= & e^{2}\left(8 \pi^{3 h}\right) \int_{-i \infty}^{+i \infty} \frac{\mathrm{d} c}{2 \pi i} f_{\delta, 0}(c) \int \widetilde{\mathrm{d}^{2} s} \Gamma\left(\frac{1+2 \Delta+c-h}{2}\right) \Gamma\left(\frac{1+2 \Delta-c-h}{2}\right) \\
& \int \prod_{i=1}^{4} \frac{\mathrm{d} t_{i}}{t_{i}} t_{i}^{\Delta_{i}} J_{1} \cdot J_{2} \exp \left[-\left(1+s^{2}\right) t_{1} t_{2} P_{12}-\left(1+\bar{s}^{2}\right) t_{1} t_{2} P_{34}-s \bar{s} \sum_{(i j)}^{\prime} t_{i} t_{j} P_{i j}\right] .
\end{aligned}
$$

where we have defined the "currents":

$$
J_{1}=t_{1} P_{1}-t_{2} P_{2}, \quad J_{2}=t_{3} P_{3}-t_{4} P_{4} .
$$


This expression is very close to the corresponding one for scalar exchange, and accordingly the rest of the calculation is now essentially the same. Using Symanzik's star formula we write the above as a Mellin amplitude,

$$
M\left(\delta_{i j}\right)=8 \gamma_{12} e^{2} \int_{-i \infty}^{+i \infty} \frac{\mathrm{d} c}{2 \pi i} f_{\delta, 0}(c) I(12, h-1, c) I(34, h-1,-c),
$$

with $\gamma_{12}=\frac{s_{13}-s_{23}}{2}$ and $I(12, h, c)$ as in (3.19), except for the crucial difference $h \rightarrow h-1$. This difference arises from the extra factors of $1 / s, 1 / \bar{s}$ in the integrals relative to the ones appearing in the Mellin amplitude for scalar exchange. These in turn appear due to the presence of the non-exponentiated $P_{13}, P_{24}, \ldots$ terms in the integrand of (6.19). After these integrals are performed we obtain

$$
M\left(\delta_{i j}\right)=4 \gamma_{12} e^{2} \int_{-i \infty}^{+i \infty} \frac{\mathrm{d} c}{2 \pi i} \frac{l_{h-1}(c) l_{h-1}(-c)}{(\delta-h)^{2}-c^{2}}
$$

with $\delta=d-1$. To evaluate the integral we simply notice that it is the same as that appearing in a scalar exchange diagram of conformal dimension $\Delta=\delta-1=d-2$ and in dimension $h \rightarrow h-1$. Therefore we can evaluate it exactly to find

$$
\begin{aligned}
M\left(s_{12}\right)= & \frac{4 \gamma_{12}}{s_{12}-(\delta-1)} \frac{e^{2} \Gamma\left(\frac{2 \Delta+\delta-h}{2}\right) \Gamma\left(\frac{2 \Delta+\delta-h}{2}\right)}{\Gamma(1+\delta-h)} \\
& { }_{3} F_{2}\left(\frac{1-2 \Delta+\delta}{2}, \frac{1-2 \Delta+\delta}{2}, \frac{(\delta-1)-s_{12}}{2} ; \frac{1+\delta-s_{12}}{2}, 1+\delta-h ; 1\right) .
\end{aligned}
$$

Alternatively, we can find the poles in $s_{12}$ by pole pinching to find their position has shifted. The result is

$$
M\left(s_{12}\right)=\sum_{n=0}^{+\infty} \frac{4 \gamma_{12}}{s_{12}-(\delta-1)-2 n} P_{n}^{\delta} \hat{V}_{[0,0, n]}^{\Delta, \Delta, \delta-1} \hat{V}_{[0,0, n]}^{\Delta, \Delta-1}
$$

where it is understood that $\delta=d-1$, and we have

$$
\begin{aligned}
\hat{V}_{[0,0,0]}^{\Delta, \Delta-1} & =e \Gamma\left(\frac{(\delta-1)+2 \Delta-2(h-1)}{2}\right), \\
\hat{V}_{\left[0,0, n_{1}\right]}^{\Delta, \Delta, \delta-1} & =\hat{V}_{[0,0,0]}^{\Delta, \Delta, \delta-1}\left(1-\frac{1}{2}[2 \Delta+(\delta-1)]+(\delta-1)\right)_{n_{1}} \\
P_{n}^{\delta} & =[n ! \Gamma(1+\delta-h+n)]^{-1} .
\end{aligned}
$$

There are several interesting things to notice in this result. For instance these are essentially the same vertices appearing in $\phi^{3}$ theory, upon shifting $h \rightarrow h-1, \delta \rightarrow \delta-1$. Also, this is an exact expression, i.e. there are no terms analytic in $s_{12}$ that we've missed, and expressions (6.23), (6.24) are identical. The main novelty is the factor of $\gamma_{12}$, whose appearance however had already been predicted by Mack [19]. It is interesting to notice that the amplitude shows factorisation, since this term is given by

$$
2 \gamma_{12}=s_{13}-s_{23}=\left(k_{1}-k_{2}\right) \cdot\left(k_{3}-k_{4}\right) \text {. }
$$




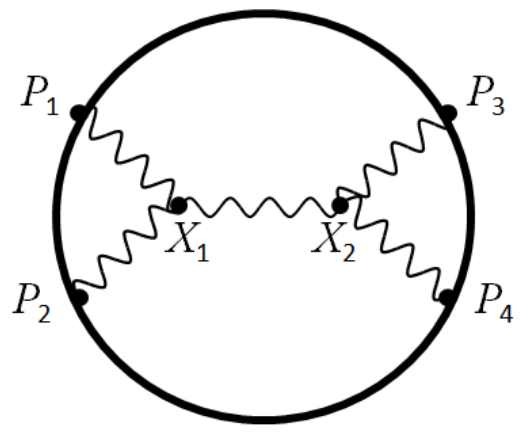

Figure 9. Current four-point function amplitude with current exchange.

More precisely, it would show exact factorisation if the $P$ 's appearing in the index structures of the three-point amplitudes $\langle J \mathcal{O O}\rangle$, could be transformed into $k$ 's. The simplicity of this result suggests that our Feynman rules can be perhaps extended to the case where there are propagating currents.

\subsection{Current 4-point amplitude}

In this section, we compute a four point function of currents using AdS/CFT. We consider non-abelian gague theory in AdS, described by an action

$$
S_{Y M}=-\int \mathrm{d}^{d+1} x \sqrt{-g} \frac{1}{4} \operatorname{Tr}\left(F_{M N} F^{M N}\right)
$$

with $F_{M N}^{a}=\partial_{M} A_{N}^{a}-\partial_{N} A_{M}^{a}+i e f^{a b c} A_{M}^{b} A_{N}^{c}$, and want to evaluate the CFT amplitude

$$
A_{4}=\left\langle J^{a, M_{1}}\left(P_{1}\right) J^{b, M_{2}}\left(P_{2}\right) J^{c, M_{3}}\left(P_{3}\right) J^{d, M_{4}}\left(P_{4}\right)\right\rangle
$$

From the action above, there are two kinds of diagrams contributing to the current four point function, a contact interaction and a current exchange diagram. The latter can occur in any of three different channels - we show the $s$-channel diagram in figure 9 . The contact interaction is elementary using our methods, since there is only an $X$ integration to perform which is trivial, and the amplitude is immediately written

$$
A_{c}=\frac{\pi^{h}}{2} \mathcal{E}_{4} \int \mathrm{d} \delta_{i j}\left(\prod_{i=1}^{4} D_{d-1}^{M_{i} A_{i}}\right) C_{4}\left[f^{a b e} f^{c d e} \eta_{A_{1} A_{3}} \eta_{A_{2} A_{4}}+\text { perms }\right] \prod_{i<j}^{4} \Gamma\left(\delta_{i j}\right)\left(P_{i j}\right)^{-\delta_{i j}}
$$

where $\sum_{i \neq j} \delta_{i j}=d-1$ and the overall constant is $C_{4}=i e^{2} \Gamma\left(\frac{3 d-4}{2}\right)$. The $D$ operators act on the products of $P_{i j}$ and are contracted with the Minkowski metrics to give the overall index structure. Notice that the integrand contains the Yang-Mills theory contact diagram in flat space. As a non-trivial check on the arguments of section 5, we show in appendix D that the result of acting with $D$ operators is indeed a polynomial in $I, X$ structures. Let us move on to the exchange diagrams. In the following we shall only consider the $s$-channel exchange, and we will denote the corresponding amplitude by $A_{s}$. As usual, the four-point function is the gluing of two three-point functions,

$$
A_{s}=\int \frac{\mathrm{d} c}{2 \pi i} f_{\delta}^{1} \int_{\partial \mathrm{AdS}} \mathrm{d} Q\left\langle J^{a, M_{1}}\left(P_{1}\right) J^{b, M_{2}}\left(P_{2}\right) J_{h+c}^{e, N}(Q)\right\rangle\left\langle J^{c, M_{1}}\left(P_{1}\right) J^{d, M_{2}}\left(P_{2}\right) J_{h-c}^{e, N}(Q)\right\rangle
$$


with

$$
\begin{gathered}
\left\langle J^{a, M_{1}}\left(P_{1}\right) J^{b, M_{2}}\left(P_{2}\right) J_{c, h+c}^{N}(Q)\right\rangle=e\left(2 \pi^{h}\right) f^{a b c} \int \frac{\mathrm{d} t_{1}}{t_{1}} \frac{\mathrm{d} t_{2}}{t_{2}} \frac{\mathrm{d} s}{s} t_{1}^{d-1} t_{2}^{d-1} s^{h+c} \\
D^{M_{1} A_{1}} D^{M_{2} A_{2}} D_{h+c}^{N A_{3}}\left[\left(t_{1} P_{1}-t_{2} P_{2}\right)_{A_{3}} \eta_{A_{1} A_{2}}+\left(t_{2} P_{2}-s Q\right)_{A_{1}} \eta_{A_{2} A_{3}}\right. \\
\left.+\left(s Q-t_{1} P_{1}\right)_{A_{2}} \eta_{A_{1} A_{3}}\right] \exp \left(-t_{1} t_{2} P_{12}+2 s\left(t_{1} P_{1}+t_{2} P_{2}\right) \cdot Q\right) .
\end{gathered}
$$

The presence of $Q$ 's in the expression, and also of $Q$ derivatives inside the $D_{h+c}$ operator complicates the calculations. Fortunately, there is a significant simplification. Recall that originally we had $D^{M A} X_{A}=0$. After the $X$ integrations are performed this means that

$$
\int\left(\prod_{i} \frac{\mathrm{d} t_{i}}{t_{i}} t_{i}^{\Delta{ }^{\Delta}}\right) D^{M A}\left(\sum t_{i} P_{i, A}\right) e^{-\sum t_{i} t_{j} P_{i j}}=0 .
$$

We interpret this as "momentum conservation". Now, the operator $D_{h+c}$ is given by

$$
D_{h+c}^{N A_{3}}=\frac{h+c-1}{h+c} \eta^{N A_{3}}+\frac{1}{h+c} \frac{\partial}{\partial Q^{N}} Q^{A_{3}} .
$$

Consider contracting the second piece of the above with each term of the second line of (6.33). The first such term leads to a vanishing result, since it is nothing but the operator $D^{M_{1} A_{1}} D_{A_{1}}^{M_{2}}$ acting on a $\left\langle J^{N} \mathcal{O O}\right\rangle$ amplitude, which vanishes when contracted with $Q^{N}$. The remaining two terms on the second line become

$$
\simeq t_{2} P_{2, A_{1}} Q_{A_{2}}-t_{1} P_{1, A_{2}} Q_{A_{1}}
$$

Using momentum conservation to trade $Q$ for $P_{1}$ and $P_{2}$ and the result is easily seen to vanish (recall that $D^{M_{i} A_{i}} P_{A_{i}}$ is vanishing). Therefore, in $D_{h+c}$ it suffices to keep its Minkowski metric part. Further, any $Q$ with a free index may be traded for $P_{1}, P_{2}$. The net result is that we have

$$
\begin{aligned}
& \left\langle J^{a, M_{1}}\left(P_{1}\right) J^{b, M_{2}}\left(P_{2}\right) J_{h+c}^{c, N}(Q)\right\rangle=e\left(2 \pi^{h}\right) f^{a b c} \int \frac{\mathrm{d} t_{1}}{t_{1}} \frac{\mathrm{d} t_{2}}{t_{2}} \frac{\mathrm{d} s}{s} t_{1}^{d-1} t_{2}^{d-1} s^{h+c} D^{M_{1} A_{1}} D^{M_{2} A_{2}} \\
& \quad \frac{h+c-1}{h+c} \eta^{N A_{3}}\left[\left(t_{1} P_{1}-t_{2} P_{2}\right)_{A_{3}} \eta_{A_{1} A_{2}}+2 t_{2} P_{2, A_{1}} \eta_{A_{2} A_{3}}-2 t_{1} P_{1, A_{2}} \eta_{A_{1} A_{3}}\right] \\
& \quad \exp \left(-t_{1} t_{2} P_{12}+2 s\left(t_{1} P_{1}+t_{2} P_{2}\right) \cdot Q\right) .
\end{aligned}
$$

Of course, a completely analogous expression holds for the other three point function appearing in (6.33). Since all the details of index structure have now decoupled from the integrals, the rest of calculation is essentially the same as that of the current exchange diagram of the previous section. The $Q$ integral is performed, and the result can be put into the form of a Mellin amplitude using Symanzik's star formula. In the end we obtain

$$
A_{s}=\frac{\pi^{h}}{2} \mathcal{E}_{4} \int \mathrm{d} \delta_{i j}\left(\prod_{i=1}^{4} D^{M_{i} A_{i}}\right) M_{A_{1}, \ldots, A_{4}}\left(\delta_{i j}\right) \prod_{i<j} \Gamma\left(\delta_{i j}\right)\left(P_{i j}\right)^{-\delta_{i j}}
$$

with

$$
\begin{aligned}
M^{A_{1} A_{2} A_{3} A_{4}}\left(s_{12}\right)= & \frac{1}{2} \frac{I^{A_{1} A_{2} A_{3} A_{4}}\left(s_{12}, \gamma_{12}\right)}{s_{12}-(d-2)} \frac{e^{2} \Gamma\left(\frac{3(d-1)-h}{2}\right)^{2}}{\Gamma\left(\frac{d}{2}\right)} \\
& { }_{3} F_{2}\left(\frac{2-d}{2}, \frac{2-d}{2}, \frac{d-2-s_{12}}{2} ; \frac{d-s_{12}}{2}, \frac{d}{2} ; 1\right) .
\end{aligned}
$$


or equivalently,

$$
M^{A_{1} \ldots A_{4}}=I^{A_{1} A_{2} A_{3} A_{4}}\left(s_{12}, \gamma_{12}\right) \sum_{n=0}^{+\infty} \frac{P_{n}^{d-1}}{s_{12}-(d-2)-2 n} \hat{V}_{[0,0, n]}^{d-1, d-1, d-2} \hat{V}_{[0,0, n]}^{d-1, d-1, d-2} .
$$

The vertices in the above are the same that appeared in (6.24) Specializing our expressions for $d=4$ we get the simple result

$$
M^{A_{1} \ldots A_{4}}=\frac{225 \pi e^{2}}{256} I^{A_{1} A_{2} A_{3} A_{4}}\left(s_{12}, \gamma_{12}\right)\left(\frac{2}{s-2}+\frac{1}{s-4}\right)
$$

We have yet to characterize the index structure $I_{A_{1} A_{2} A_{3} A_{4}}$. It is the result of contracting two currents of the form

$$
J_{A_{i} A_{j} A_{k}} \equiv\left(t_{i} P_{i}-t_{j} P_{j}\right)_{A_{k}} \eta_{A_{i} A_{j}}+2 t_{j} P_{j, A_{i}} \eta_{A_{j} A_{k}}-2 t_{i} P_{i, A_{j}} \eta_{A_{i} A_{k}}
$$

followed by $t_{i} t_{j} P_{i j} \rightarrow \frac{\delta_{i j}}{P_{i j}}$. Doing this we obtain

$$
\begin{aligned}
I^{A_{1} A_{2} A_{3} A_{4}}= & 4 \gamma_{12} \eta^{A_{1} A_{2}} \eta^{A_{3} A_{4}} \\
& -4\left[\frac{\left(\gamma_{12}-s_{12}\right)}{2 P_{13}}\left(\eta^{A_{3} A_{4}} P_{1}^{A_{2}} P_{3}^{A_{1}}+\eta^{A_{1} A_{2}} P_{1}^{A_{3}} P_{3}^{A_{4}}-2 \eta^{A_{1} A_{3}} P_{1}^{A_{2}} P_{3}^{A_{4}}\right)\right. \\
& -(1 \leftrightarrow 2)-(3 \leftrightarrow 4)+(1 \leftrightarrow 2,3 \leftrightarrow 4)] .
\end{aligned}
$$

It is clear that if one identifies $P_{i}$ with a fictional momentum $k_{i}$, then the index structure of this expression roughly corresponds to the one appearing in the analogous diagram for Yang-Mills theory in flat space. To obtain the full conformal index structure we have to act with the $D$ operators. This is most simply performed with the aid of a computer. ${ }^{7}$ The result is too long to be presented here, but we have been able to check that it is simply a polynomial in the $X_{i j}^{M}$ and $I^{M N}$ structures introduced in (1.16), (1.17), as expected from our general arguments in section 5 .

Importantly the propagator/vertex structure remains, and it is exactly the same as what we have computed in the scalar four point function current exchange diagram. In this sense, that computation already contains all the dynamic information relevant for the four-current correlator. What the current result shows is that it is possible to quite simply decouple the details of the index structure from the rest of the calculation.

\section{Discussion and outlook}

In this paper we have showed how calculations of correlation functions in AdS/CFT are significantly made simpler by the combined use of the embedding formalism and the Mellin representation. The embedding formalism essentially makes the kinematic AdS integrals become trivial, at the expense of introducing integrations in Schwinger parameters. At this point the Mellin representation becomes useful by translating such integrations to Mellin

\footnotetext{
${ }^{7}$ Notebooks are available upon request.
} 
space via Symanzik's formula. With these methods we have managed to write down four point Mellin amplitudes explicitly in terms of hypergeometric functions. For higher point amplitudes, we have shown how there seems to be a set of Feynman rules which allows us to write them down. Although we have not proved in full generality that these rules are correct, we have presented non-trivial evidence in the form of the explicit calculation of higher point amplitudes.

The similarity between Mellin amplitudes and flat space scattering amplitudes had been noticed already in [21]. There it was conjectured that in the high energy limit where the $\delta_{i j}$ parameters become large, the Mellin amplitude reduces to a flat-space amplitude of massless particles. In this sense, AdS space can be thought of as naturally providing an IR cut-off for flat-space amplitudes. As far as we have been able to check, the results we have derived in this paper agree with the proposal of [21], at least in the scalar sector. When free indices are present, we are faced with difficulties, as the Mellin amplitude now depends on the coordinates $P$ as well as on the Mandelstam invariants. Our results suggest that we should identify the corresponding flat space amplitude with the reduced Mellin amplitude, i.e. the amplitude obtained before acting with the $D$ operators. Indeed, as we've pointed out throughout this paper, those amplitudes are remarkable similar to flat space amplitudes, if one identifies the coordinates $P$ with momenta $k$.

We clearly lack a deeper understanding of the structure of general Mellin amplitudes, such as pole structure, relation to lower point amplitudes and unitarity properties. ${ }^{8}$ Presumably such an understanding could lead to a proof of our proposed Feynman rules for Mellin amplitudes in scalar theory. It could also help us to understand the structure of amplitudes involving fields with spin, and if whether Feynman rules can be written down in this case. As a first easy check one should compute higher $n$-point functions of scalars with gauge fields propagating in the internal lines.

An obvious continuation of our work is the investigation of loop amplitudes. These were first discussed in [21], but there it was not attempted to write the result $\grave{a}$ la Feynman. It would be interesting to check whether our rules for tree-level scalar amplitudes generalize to loop amplitudes in the expected way. Although in our formalism one would never obtain loop momenta integrals, one does obtain Mellin-Barnes type integrals, which roughly correspond to integrals over conformal dimension. Since the Mellin momenta $k_{i}$ square to conformal dimension, perhaps these integrals can be interpreted as integrals over the norm of the loop momenta.

Recently there was an attempt to use the spinor-helicity formalism to compactly describe CFT correlators in momentum space [35]. Our methods allow for a different tack on the same problem: since the embedding formalism allows us to describe the index structure of Mellin amplitudes in terms of $d+2$ vectors $P$ satisfying $P^{2}=0$, use of spinor-helicity formalism suggests itself. For instance one could to use the six-dimensional formalism of [36] to describe four-dimensional conformal field theory amplitudes. Curiously, for $d=2$ it seems that the \pm helicities of four dimensional massless particles map to (anti)holomorphic two-dimensional amplitudes. This is possible because after the action of $D$ operators, the

\footnotetext{
${ }^{8}$ For a proposed BCFW type recursion relation for Witten diagrams see [33, 34].
} 
conformal index structure of a CFT amplitude resembles that of a flat-space amplitude with higher dimension operators: the current 3-pt function has contributions cubic in $P$, which would come from an $\left(F_{a b}\right)^{3}$ term in four dimensions.

It seems likely that the calculation of the stress-tensor four-point function should be achievable using our methods. The results we have obtained in this paper for the current four-point function lead us to expect that the index structure should decouple from the exchange part of the amplitude. The latter should essentially be the same as that obtained as for stress-tensor exchange in scalar theory. The full amplitude will be obtained by acting with four $D_{2}$ operators on the reduced Mellin amplitude, which should have an index structure similar to a four-graviton flat-space amplitude upon identification of the momentum with the coordinate $P$. We hope to present more on this and other stress-tensor correlation functions elsewhere [37].

Finally, we have seen that there seems to be an intriguing connection between the correlation functions we have been computing for $d$-dimensional CFT's, and a theory of massless particles in $d+2$ dimensions. The connection is given by interpreting boundary point of the CFT as $d+2$ null vectors $P$, which can then be interpreted as momenta. It is highly suggestive that we were able to write down the relations (5.3) and even a "momentum conservation" equation (6.4). It would be interesting to see if this connection can be developed further.

\section{Acknowledgments}

It is a pleasure to acknowledge discussions with Atish Dabholkar, Paolo Benincasa, Eduardo Conde and Xiàn Camanho. The author would like to thank the University of Santiago de Compostela, where part of this work was performed, for funding and hospitality. The author acknowledges funding from the LPTHE, Université Pierre et Marie Curie, and partial support from the Portuguese FCT funded project CERN/FP/116377/2010.

\section{A Some integrals}

In this section we describe the computation of the AdS and AdS boundary integrals which appear throughout the paper. These calculations have appeared already in [21], and we include them here for completeness. The first such calculation is the proof that

$$
\int_{0}^{+\infty} \prod_{i}\left(\frac{\mathrm{d} t_{i}}{t_{i}} t^{\alpha_{i}}\right) \int_{\mathrm{AdS}} \mathrm{d} X e^{2 T \cdot X}=\pi^{h} \Gamma\left(\frac{\sum_{i} \alpha_{i}-2 h}{2}\right) \int_{0}^{+\infty} \prod_{i}\left(\frac{\mathrm{d} t_{i}}{t_{i}} t^{\alpha_{i}}\right) e^{T^{2}} .
$$

with $T=\sum t_{i} P_{i}$. We proceed by computing the left-hand side. First we evaluate the AdS integral. By Lorentz invariance we can consider the case where $T=|T|(1,1,0)$. We also parameterize $A d S_{d+1}$ space by

$$
X=\left(X^{+}, X^{-}, X^{\mu}\right)=\frac{1}{x_{0}}\left(1, x_{0}^{2}+x^{2}, x^{\mu}\right)
$$


and define $h \equiv d / 2$. Then we get

$$
\begin{aligned}
\int_{\mathrm{AdS}} \mathrm{d} X e^{2 T \cdot X} & =\int_{0}^{+\infty} \frac{\mathrm{d} x_{0}}{x_{0}} x_{0}^{-d} \int_{0}^{+\infty} \mathrm{d}^{d} x e^{-\left(1+x_{0}^{2}+x^{2}\right)|T| / x_{0}} \\
& =\pi^{h} \int_{0}^{+\infty} \frac{\mathrm{d} x_{0}}{x_{0}} x_{0}^{-h} e^{-x_{0}+T^{2} / x_{0}}
\end{aligned}
$$

The original integral becomes

$$
\begin{aligned}
& \pi^{h} \int_{0}^{+\infty} \prod_{i}\left(\frac{\mathrm{d} t_{i}}{t_{i}} t^{\alpha_{i}}\right) \int_{0}^{+\infty} \frac{\mathrm{d} x_{0}}{x_{0}} x_{0}^{-h} e^{-x_{0}+\left(\sum_{i} t_{i} P_{i}\right)^{2} / x_{0}}= \\
& \quad=\pi^{h} \int_{0}^{+\infty} \prod_{i}\left(\frac{\mathrm{d} t_{i}}{t_{i}} t^{\alpha_{i}}\right) e^{T^{2}} \int_{0}^{+\infty} \frac{\mathrm{d} x_{0}}{x_{0}} x_{0}^{\sum_{i} \alpha_{i} / 2-h} e^{-x_{0}}= \\
& \quad=\pi^{h} \Gamma\left(\frac{\sum_{i} \alpha_{i}-2 h}{2}\right) \int_{0}^{+\infty} \prod_{i}\left(\frac{\mathrm{d} t_{i}}{t_{i}} t^{\alpha_{i}}\right) e^{T^{2}}
\end{aligned}
$$

where in the second step we rescaled $t_{i} \rightarrow t_{i} / \sqrt{x_{0}}$

Next we prove:

$$
\int_{0}^{+\infty} \frac{\mathrm{d} s}{s} \frac{\mathrm{d} \bar{s}}{\bar{s}} s^{h+c} s^{h-c} \int_{\partial \mathrm{AdS}} \mathrm{d} Q e^{2 T \cdot Q}=2 \pi^{h} \int_{0}^{+\infty} \frac{\mathrm{d} s}{s} \frac{\mathrm{d} \bar{s}}{\bar{s}} s^{h+c} s^{h-c} e^{T^{2}}
$$

with $T \equiv(s X+\bar{s} Y)$. First we evaluate the boundary integral on the left-hand side. Using the parameterization

$$
Q=\left(Q^{+}, Q^{-}, Q^{\mu}\right)=\left(1, x^{2}, x^{\mu}\right)
$$

we find

$$
\int_{\partial \operatorname{AdS}} \mathrm{d} Q e^{2 T \cdot Q}=\int_{0}^{+\infty} \mathrm{d}^{d} x e^{-|T|\left(1+x^{2}\right)}=\frac{\pi^{h}}{|T|^{h}} e^{-|T|} .
$$

Now, noticing that $1=\int_{0}^{+\infty} \mathrm{d} v \delta(v-s-\bar{s})$, we find

$$
\begin{aligned}
\int_{0}^{+\infty} & \frac{\mathrm{d} s}{s} \frac{\mathrm{d} \bar{s}}{\bar{s}} s^{h+c} s^{h-c} \frac{\pi^{h}}{|T|^{h}} e^{-|T|}= \\
& =\int_{0}^{+\infty} \mathrm{d} v \int_{0}^{+\infty} \frac{\mathrm{d} s}{s} \frac{\mathrm{d} \bar{s}}{\bar{s}} s^{h+c} s^{h-c} \delta(v-s-\bar{s}) \frac{\pi^{h}}{|s X+\bar{s} Y|^{h}} e^{-|s X+\bar{s} Y|} \\
& =\pi^{h} \int_{0}^{+\infty} \frac{\mathrm{d} v}{v} \int_{0}^{+\infty} \frac{\mathrm{d} s}{s} \frac{\mathrm{d} \bar{s}}{\bar{s}} s^{h+c} s^{h-c} \delta(1-s-\bar{s}) \frac{v^{h}}{|s X+\bar{s} Y|^{h}} e^{-|s X+\bar{s} Y|} \\
& =\pi^{h} \int_{0}^{+\infty} \frac{\mathrm{d} v}{v} \int_{0}^{+\infty} \frac{\mathrm{d} s}{s} \frac{\mathrm{d} \bar{s}}{\bar{s}} s^{h+c} s^{h-c} \delta(1-s-\bar{s}) v^{h} e^{v(s X+\bar{s} Y)^{2}}
\end{aligned}
$$

Finally rescaling $s \rightarrow s / \sqrt{v}, \bar{s} \rightarrow \bar{s} / \sqrt{v}$ the $v$ integral is performed and we find the righthand side of (A.5), as promised.

\section{B The Symanzik star formula}

For completeness, in this section we review the Symanzik star integration formula in Euclidean space as discussed in [19]. For a proof and more details we refer the reader to the 
original reference [22]. Consider a set of $n$ points in Euclidean space $x_{i}$ and their differences $x_{i}-x_{j}$. In the embedding formalism we have $P_{i j} \equiv-2 P_{i} \cdot P_{j}=\left(x_{i}-x_{j}\right)^{2}$. Then Symanzik's formula is:

$$
\int_{0}^{+\infty}\left(\prod_{i=1}^{n} \frac{\mathrm{d} t_{i}}{t_{i}} t^{\Delta_{i}}\right) e^{-\left(\sum_{1 \leq i<j \leq n} t_{i} t_{j} P_{i j}\right)}=\frac{\pi^{h} / 2}{(2 \pi i)^{\frac{1}{2} n(n-3)}} \int \mathrm{d} \delta_{i j} \prod_{1 \leq i<j \leq n} \Gamma\left(\delta_{i j}\right)\left(P_{i j}\right)^{-\delta_{i j}}
$$

The integration measure on the right-hand side deserves further explanation. The parameters $\delta_{i j}$, symmetric in $i, j$, satisfy

$$
\sum_{i \neq j} \delta_{i j}=\Delta_{j}
$$

for all $i$. Now pick a particular solution of the set of equations (B.2), $\delta_{i j}^{0}$. Then we write

$$
\delta_{i j}=\delta_{i j}^{0}+\sum_{k=1}^{\frac{1}{2} n(n-3)} c_{i j, k} s_{k}
$$

with

$$
c_{i i, k}=0, \quad \sum_{j \neq i} c_{i j, k}=0
$$

Choosing as independent coefficients the $\left(\frac{1}{2} n(n-3)\right)^{2}$ coefficient $c_{i j, k}$ with $2 \leq i<j \leq n$ (with the exception of $c_{23, k}$ ), with the further restriction $\left|\operatorname{det} c_{i j, k}\right|=1$, we can write

$$
\int \mathrm{d} \delta_{i j} \rightarrow \int_{-i \infty} \prod_{k=1}^{\frac{1}{2} n(n-3)} \frac{\mathrm{d} s_{k}}{2 \pi i}
$$

The integration paths are chosen parallel to the imaginary axis, with real parts such that the real parts of the arguments of the gamma functions are positive.

\section{Details on the calculation of the six-point amplitude}

In the computation of the six point amplitude, or indeed of any amplitude involving internal lines, we have to perform boundary integrals over the coordinates $Q_{i}$ of each internal line. Since these integrals have to be done in a certain order, this breaks the symmetry of the expressions and the result seems more complicated than it is. A typical example of this is what happens in going from the third line of (4.5) to the simpler looking (4.8). In the calculation of the six- and twelve-point functions the same thing occurs. In this section we give some details on the changes of variables required to obtain a simpler looking integral for the case of the six-point amplitude. Details on the 12-point amplitude are quite technical and can be obtained upon request.

We have six integrals that can be performed, over parameters $s_{i}, \bar{s}_{i}, i=1, \ldots 3$. After the $s_{i}$ integrations are performed, then if the boundary integrations were done in the order 
$Q_{1}, Q_{2}, Q_{3}$ the integral over the $\bar{s}_{i}$ is of the form

$$
\begin{aligned}
& \int_{0}^{+\infty} \prod_{i=1}^{3}\left(\frac{\mathrm{d} \bar{s}_{i}}{\bar{s}_{i}}\right)\left(\bar{s}_{1}^{2}+1\right)^{\frac{1}{2}\left(s_{12}-s_{34}-s_{56}\right)}\left(\left(\bar{s}_{1}^{2}+1\right) \bar{s}_{2}^{2}+1\right)^{\frac{1}{2}\left(-s_{12}+s_{34}-s_{56}\right)} \\
& \quad \times\left(\bar{s}_{1}^{2}\left(\bar{s}_{3}^{2}\left(\left(\bar{s}_{1}^{2}+1\right) \bar{s}_{2}^{2}+1\right)^{2}+\bar{s}_{2}^{2}\right)+1\right)^{\frac{1}{2}\left(-c_{1}-h+s_{12}\right)}\left(\left(\bar{s}_{1}^{2}+1\right)^{2} \bar{s}_{2}^{2} \bar{s}_{3}^{2}+1\right)^{\frac{1}{2}\left(-c_{2}-h+s_{34}\right)} \\
& \quad \times\left(\left(\bar{s}_{1}^{2}+1\right) \bar{s}_{3}^{2}\left(\left(\bar{s}_{1}^{2}+1\right) \bar{s}_{2}^{2}+1\right)+1\right)^{\frac{1}{2}\left(-s_{12}-s_{34}+s_{56}\right)}
\end{aligned}
$$

which looks quite complicated. However, performing the change of variables

$$
\bar{s}_{1} \rightarrow \sqrt{x}, \quad \bar{s}_{2} \rightarrow \sqrt{y}, \quad \bar{s}_{3} \rightarrow \sqrt{z}
$$

followed by the sequence of variable changes

$$
\begin{array}{ll}
y \rightarrow \frac{y}{1+x}, & z \rightarrow \frac{z}{1+x}, \\
x \rightarrow \frac{x}{(1+y)(1+z)}, & y \rightarrow \frac{y}{1+z},
\end{array}
$$

finally leads to

$$
\int_{0}^{+\infty} \frac{\mathrm{d} x}{x} \frac{\mathrm{d} y}{y} \frac{\mathrm{d} z}{z} x^{a} y^{b} z^{c}(1+x)^{d}(1+y)^{e}(1+z)^{f}(1+x+y+z)^{g}
$$

with $g=\frac{1}{2}\left(c_{1}+c_{2}+c_{3}-h\right)$ and

$$
\begin{aligned}
& a=\frac{1}{2}\left(-c_{1}+h-s_{12}\right), \quad b=\frac{1}{2}\left(-c_{2}+h-s_{34}\right) \quad c=\frac{1}{2}\left(-c_{3}+h-s_{56}\right) \\
& d=\frac{1}{2}\left(-c_{1}-h+s_{12}\right), \quad e=\frac{1}{2}\left(-c_{2}-h+s_{34}\right), \quad f=\frac{1}{2}\left(-c_{3}-h+s_{56}\right) \text {. }
\end{aligned}
$$

\section{Index structure of current four-point function contact diagram}

We wish to evaluate:

$$
D^{M_{1} A_{1}} D^{M_{2} A_{2}} D^{M_{3} A_{3}} D^{M_{4} A_{4}}\left(\eta_{A_{1} A_{3}} \eta_{A_{2} A_{4}} \prod_{i<j}\left(P_{i j}\right)^{-\delta_{i j}}\right) .
$$

Defining the quantities

$$
\begin{aligned}
Z^{M_{1}} & \equiv s_{12}\left(X_{23}^{M_{1}}+X_{24}^{M_{1}}\right)+\gamma_{12} X_{34}^{M_{1}} \\
Z^{M_{2}} & \equiv s_{12}\left(X_{13}^{M_{2}}+X_{14}^{M_{2}}\right)-\gamma_{12} X_{34}^{M_{2}} \\
Z^{M_{3}} & \equiv s_{12}\left(X_{14}^{M_{3}}+X_{24}^{M_{3}}\right)-\gamma_{12} X_{12}^{M_{3}} \\
Z^{M_{4}} & \equiv s_{12}\left(X_{13}^{M_{4}}+X_{23}^{M_{4}}\right)+\gamma_{12} X_{12}^{M_{4}} \\
\hat{I}^{M_{i} M_{j}} & \equiv 16 \delta_{i j} \frac{I^{M_{i} M_{j}}}{P_{i} \cdot P_{j}}
\end{aligned}
$$


the result is

$$
\begin{aligned}
& 256(d-1)^{4}\left(P_{i j}\right)^{\delta_{i j}}\left(D^{M_{1} A_{1}} D^{M_{2} A_{2}} D^{M_{3} A_{3}} D^{M_{4} A_{4}} \eta_{A_{1} A_{3}} \eta_{A_{2} A_{4}} \prod_{i<j}\left(P_{i j}\right)^{-\delta_{i j}}\right)= \\
& \quad Z^{M_{1}} Z^{M_{2}} Z^{M_{3}} Z^{M_{4}}+\left[\frac{(d-1)^{2}-\delta_{12}}{\delta_{12}}\right]^{2} \hat{I}^{M_{1} M_{2}} \hat{I}^{M_{3}, M_{4}}+\hat{I}^{M_{1} M_{3}} \hat{I}^{M_{2} M_{4}}+\hat{I}^{M_{1} M_{4}} \hat{I}^{M_{2} M_{3}} \\
& \quad+\left[\frac{(d-1)^{2}-\delta_{12}}{\delta_{12}}\right]\left(Z^{M_{3} M_{4}} \hat{I}^{M_{1} M_{2}}+Z^{M_{1} M_{2}} \hat{I}^{M_{3} M_{4}}\right) \\
& \quad+\left(Z^{M_{1} M_{3}} \hat{I}^{M_{2} M_{4}}+Z^{M_{1} M_{4}} \hat{I}^{M_{2} M_{3}}+Z^{M_{2} M_{3}} \hat{I}^{M_{1} M_{4}}+Z^{M_{2} M_{4}} \hat{I}^{M_{1} M_{3}}\right)
\end{aligned}
$$

in exact agreement with the expectations of section 5 .

Open Access. This article is distributed under the terms of the Creative Commons Attribution Noncommercial License which permits any noncommercial use, distribution, and reproduction in any medium, provided the original author(s) and source are credited.

\section{References}

[1] E. Witten, Anti-de Sitter space and holography, Adv. Theor. Math. Phys. 2 (1998) 253 [hep-th/9802150] [SPIRES].

[2] S.S. Gubser, I.R. Klebanov and A.M. Polyakov, Gauge theory correlators from non-critical string theory, Phys. Lett. B 428 (1998) 105 [hep-th/9802109] [SPIRES].

[3] J.M. Maldacena, The large- $N$ limit of superconformal field theories and supergravity, Int. J. Theor. Phys. 38 (1999) 1113 [Adv. Theor. Math. Phys. 2 (1998) 231] [hep-th/9711200] [SPIRES].

[4] O. Aharony, S.S. Gubser, J.M. Maldacena, H. Ooguri and Y. Oz, Large-N field theories, string theory and gravity, Phys. Rept. 323 (2000) 183 [hep-th/9905111] [SPIRES].

[5] D.Z. Freedman, S.D. Mathur, A. Matusis and L. Rastelli, Correlation functions in the CFT $(d) / A d S(d+1)$ correspondence, Nucl. Phys. B 546 (1999) 96 [hep-th/9804058] [SPIRES].

[6] D.Z. Freedman, S.D. Mathur, A. Matusis and L. Rastelli, Comments on 4-point functions in the CFT/AdS correspondence, Phys. Lett. B 452 (1999) 61 [hep-th/9808006] [SPIRES].

[7] E. D'Hoker, D.Z. Freedman, S.D. Mathur, A. Matusis and L. Rastelli, Graviton and gauge boson propagators in AdS(d+1), Nucl. Phys. B 562 (1999) 330 [hep-th/9902042] [SPIRES].

[8] E. D'Hoker, D.Z. Freedman and L. Rastelli, AdS/CFT 4-point functions: How to succeed at z-integrals without really trying, Nucl. Phys. B 562 (1999) 395 [hep-th/9905049] [SPIRES].

[9] J. Bartels, J. Kotanski, A.M. Mischler and V. Schomerus, R-current six-point correlators in $A d S_{5}$ Supergravity, Nucl. Phys. B 832 (2010) 382 [arXiv:0912.3072] [SPIRES].

[10] J. Bartels, J. Kotanski, A.M. Mischler and V. Schomerus, Regge limit of R-current correlators in AdS Supergravity, Nucl. Phys. B 830 (2010) 153 [arXiv:0908.2301] [SPIRES].

[11] Y. Hatta and T. Ueda, Soft photon anomaly and gauge/string duality, Nucl. Phys. B 837 (2010) 22 [arXiv: 1002.3452] [SPIRES]. 
[12] E. D'Hoker, D.Z. Freedman, S.D. Mathur, A. Matusis and L. Rastelli, Graviton exchange and complete 4-point functions in the AdS/CFT correspondence, Nucl. Phys. B 562 (1999) 353 [hep-th/9903196] [SPIRES].

[13] L.I. Uruchurtu, AdS/CFT for Four-Point Amplitudes involving Gravitino Exchange, JHEP 09 (2007) 086 [arXiv: 0707.0424] [SPIRES].

[14] L.I. Uruchurtu, Four-point correlators with higher weight superconformal primaries in the AdS/CFT Correspondence, JHEP 03 (2009) 133 [arXiv:0811.2320] [SPIRES].

[15] L.I. Uruchurtu, Next-next-to-extremal Four Point Functions of $N=41 / 2$ BPS Operators in the AdS/CFT Correspondence, JHEP 08 (2011) 133 [arXiv:1106.0630] [SPIRES].

[16] G. Arutyunov and S. Frolov, Three-point Green function of the stress-energy tensor in the AdS/CFT correspondence, Phys. Rev. D 60 (1999) 026004 [hep-th/9901121] [SPIRES].

[17] E.J. Schreier, Conformal symmetry and three-point functions, Phys. Rev. D 3 (1971) 980 [SPIRES].

[18] H. Osborn and A.C. Petkou, Implications of Conformal Invariance in Field Theories for General Dimensions, Ann. Phys. 231 (1994) 311 [hep-th/9307010] [SPIRES].

[19] G. Mack, D-independent representation of Conformal Field Theories in D dimensions via transformation to auxiliary Dual Resonance Models. Scalar amplitudes, arXiv:0907.2407 [SPIRES].

[20] G. Mack, D-dimensional Conformal Field Theories with anomalous dimensions as Dual Resonance Models, arXiv:0909.1024 [SPIRES].

[21] J. Penedones, Writing CFT correlation functions as AdS scattering amplitudes, JHEP 03 (2011) 025 [arXiv: 1011.1485] [SPIRES].

[22] K. Symanzik, On Calculations in conformal invariant field theories, Lett. Nuovo Cim. 3 (1972) 734 [SPIRES].

[23] P.A.M. Dirac, Wave equations in conformal space, Annals Math. 37 (1936) 429 [SPIRES].

[24] S. Weinberg, Six-dimensional Methods for Four-dimensional Conformal Field Theories, Phys. Rev. D 82 (2010) 045031 [arXiv: 1006.3480] [SPIRES].

[25] J. Penedones, High Energy Scattering in the AdS/CFT Correspondence, arXiv:0712.0802 [SPIRES].

[26] G. Lauricella, Sulla funzioni ipergeometriche a piú variabili in Italian, Rend. Circ. Math. Palermo 7 (1893) 111.

[27] H.M. Srivastava and P.W. Karlsson, Multiple Gaussian Hypergeometric Series, Ellis Horwood, Chichester U.K. (1985).

[28] R.M. Aarts, Lauricella Functions, from MathWorld-A Wolfram Web Resource, created by E.W. Weisstein (2011), http://mathworld.wolfram.com/LauricellaFunctions.html.

[29] A.L. Fitzpatrick, J. Kaplan, J. Penedones, S. Raju, and B. van Rees, A Natural Language for AdS/CFT Correlators, arXiv:1107.1499 [SPIRES].

[30] L. Cornalba, M.S. Costa and J. Penedones, Deep Inelastic Scattering in Conformal QCD, JHEP 03 (2010) 133 [arXiv:0911.0043] [SPIRES].

[31] R. Britto, F. Cachazo, B. Feng and E. Witten, Direct Proof Of Tree-Level Recursion Relation In Yang-Mills Theory, Phys. Rev. Lett. 94 (2005) 181602 [hep-th/0501052] [SPIRES]. 
[32] I. Balitsky, Mellin representation of the graviton bulk-to-bulk propagator in AdS, Phys. Rev. D 83 (2011) 087901 [arXiv:1102.0577] [SPIRES].

[33] S. Raju, Generalized Recursion Relations for Correlators in the Gauge-Gravity Correspondence, Phys. Rev. Lett. 106 (2011) 091601 [arXiv:1011.0780] [SPIRES].

[34] S. Raju, Recursion Relations for AdS/CFT Correlators, Phys. Rev. D 83 (2011) 126002 [arXiv: 1102.4724] [SPIRES].

[35] J.M. Maldacena and G.L. Pimentel, On graviton non-Gaussianities during inflation, JHEP 09 (2011) 045 [arXiv: 1104.2846] [SPIRES].

[36] C. Cheung and D. O'Connell, Amplitudes and Spinor-Helicity in Six Dimensions, JHEP 07 (2009) 075 [arXiv: 0902.0981] [SPIRES].

[37] M.F. Paulos, to appear. 